Article

\title{
Synthesis and Bioactivities of Novel Pyrazole Oxime Derivatives Containing a 5-Trifluoromethylpyridyl Moiety
}

\author{
Hong Dai ${ }^{1}$, Jia Chen ${ }^{1}$, Hong $\mathrm{Li}^{2}$, Baojiang Dai ${ }^{3}$, Haibing He ${ }^{1, *}$, Yuan Fang ${ }^{1}$ and Yujun Shi ${ }^{1, *}$ \\ 1 College of Chemistry and Chemical Engineering, Nantong University, Nantong 226019, China; \\ daihong_2015@aliyun.com (H.D.); 15642891665@163.com (J.C.); fyuan6586@aliyun.com (Y.F.) \\ 2 Nantong Fengtian Chemical Industry Co. Ltd., Nantong 226005, China; leehomer@sina.com \\ 3 School of Chemical and Biological Engineering, Nantong Vocational University, Nantong 226007, China; \\ dbj20110802@163.com \\ * Correspondence: gaohbhe2015@aliyun.com (H.H.); syj@ntu.edu.cn (Y.S.); \\ Tel./Fax: +86-513-8501-2851 (H.H. \& Y.S.)
}

Academic Editor: Derek J. McPhee

Received: 1 February 2016; Accepted: 23 February 2016; Published: 27 February 2016

\begin{abstract}
In this study, in order to find novel biologically active pyrazole oxime compounds, a series of pyrazole oxime derivatives containing a 5-trifluoromethylpyridyl moiety were synthesized. Preliminary bioassays indicated that most title compounds were found to display good to excellent acaricidal activity against Tetranychus cinnabarinus at a concentration of $200 \mu \mathrm{g} / \mathrm{mL}$, and some designed compounds still showed excellent acaricidal activity against Tetranychus cinnabarinus at the concentration of $10 \mu \mathrm{g} / \mathrm{mL}$, especially since the inhibition rates of compounds $\mathbf{8 e}, \mathbf{8 f}, \mathbf{8 1}$, $\mathbf{8 m}, \mathbf{8 n}, \mathbf{8 p}$, and $\mathbf{8 q}$ were all $100.00 \%$. Interestingly, some target compounds exhibited moderate to good insecticidal activities against Plutella xylostella and Aphis craccivora at a concentration of $200 \mu \mathrm{g} / \mathrm{mL}$; furthermore, compounds $\mathbf{8 e}$ and $\mathbf{8 1}$ possessed outstanding insecticidal activities against Plutella xylostella under the concentration of $50 \mu \mathrm{g} / \mathrm{mL}$.
\end{abstract}

Keywords: pyrazole oxime; 5-trifluoromethylpyridyl; synthesis; bioactivity

\section{Introduction}

Agrochemicals, together with genetically modified insect-resistant crops and biological plant protection methods, prevent severe harvest losses caused by phytophagous insects and mites [1-3]. Over the past decades, a great variety of chemical pesticides have been developed and used in the protection of crops, among which pyrazole derivatives occupy a considerable proportion in insecticides, germicides and acaricides [4-7]. As a well-known pyrazole-based pesticide developed by Nihon Nohyaku Co. in 1991, Fenpyroximate (Figure 1) has been widely used in protecting various crops due to its high efficiency against agricultural mites such as Polyphagotarsonemus latus Banks and Tetranychus urticae Koch, and its low toxicity to mammals [8]. Unfortunately, continuous application of Fenpyroximate in recent years led to the occurrence of resistance from some field populations of T. urticae [9]. Researchers are therefore propelled to search for new compounds that are highly active against phytophagous mites, and the development of novel analogues of Fenpyroximate is extraordinarily focused on [10-12].

Fenpyroximate is structurally characterized by the unique 4-pyrazole oxime, which is recognized as an irreplaceable pharmacophore for acaricidal or insecticidal activities. In the process of developing new Fenpyroximate derivatives, modification of phenyl B (Figure 1) and its substituents was mainly concentrated on with the 4-pyrazole oxime moiety retained. For example, Dai and coworkers reported some acaricidal or insecticidal Fenpyroximate mimics that were obtained by replacing the phenyl B (Figure 1) with thiazole [13], or a pyridyl group [14]. Many of these efforts succeeded in getting 
some heterocycle-based pyrazole oxime compounds that displayed promising biological activities. Therefore, structural optimization of the phenyl B of Fenpyroximate (Figure 1) to explore novel bioactive molecules is a reasonable design.

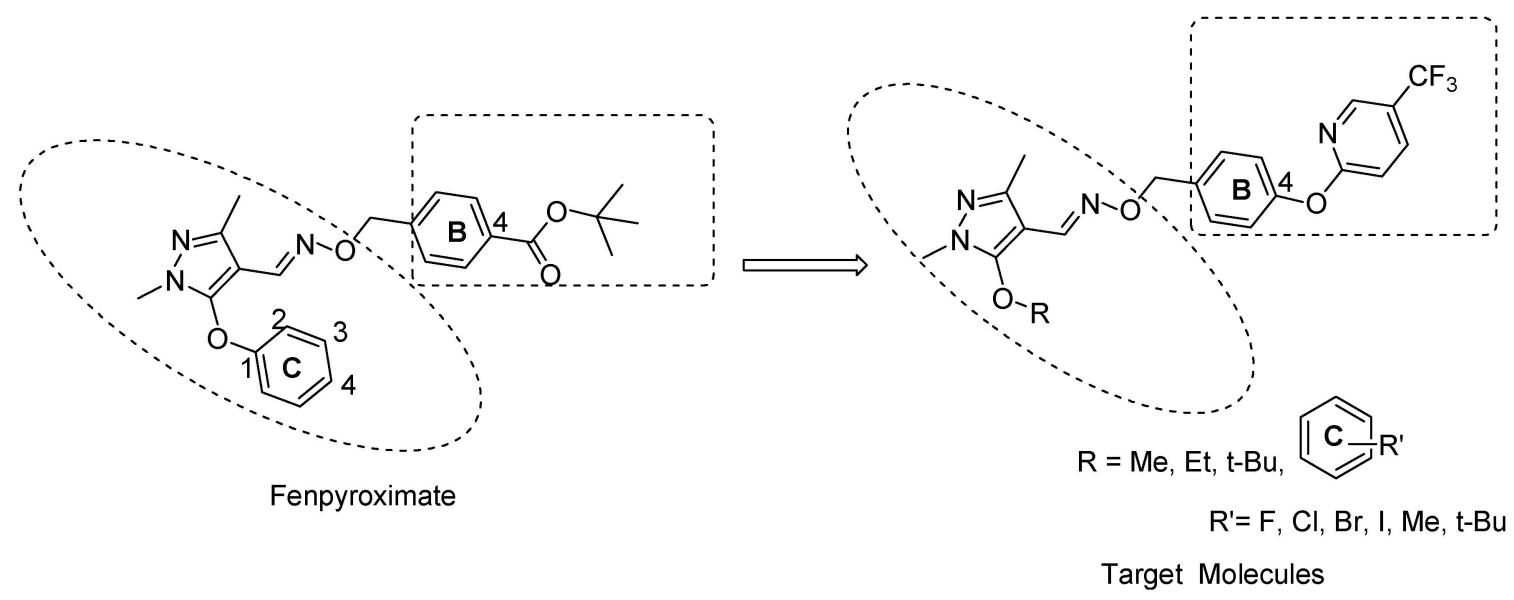

Figure 1. The design of the target molecules.

As we know, due to its special aromaticity, basicity and hydrophilicity, a pyridyl group is a very commonly used active moiety in pesticidal molecules [15-21]. In addition, many investigations have indicated that introducing $\mathrm{CF}_{3}$ into heterocyclic molecules mostly results in the improvement of physical, chemical and biological properties [22,23]. In fact, it has been proved that $\mathrm{CF}_{3}$-substituted pyridine is a highly efficient functional group in active pesticidal molecules, for instance neonicotinoids insecticide Sulfoxaflor [24], and dichloropropene insecticide Pyridalyl [25], each of which has a similar trifluoromethyl pyridyl fragment.

Encouraged by these reports, in this study we integrated the 5-trifluoromethyl pyridyl unit into the scaffold of Fenpyroximate for the first time, and the substituents on the 5-position of the pyrazole ring are screened and optimized (Figure 1). Insecticidal and acaricidal activities of the target compounds were tested accordingly.

\section{Results and Discussion}

\subsection{Chemistry}

As shown in Scheme 1, 23 pyrazole oxime derivatives bearing a 5-trifluoromethyl pyridyl moiety were conveniently synthesized. The synthon 4 was synthesized from commercially available 2-chloro-5-trifluoromethylpyridine (1) as shown in Scheme 1. Compound 1 reacted with 4-hydroxybenzaldehyde using $\mathrm{Cs}_{2} \mathrm{CO}_{3}$ as the base and $N, N$-dimethylformamide as the solvent to give intermediate 2 in 75\% yield. The next reaction with $\mathrm{NaBH}_{4}$ afforded compound 3 in $90 \%$ yield. Further chlorination of compound 3 obtained intermediate 4 in satisfactory yield. The addition of a few drops of $N, N$-dimethylformamide accelerated the chlorination reaction. The pyrazole oximes $\mathbf{7 a - 7} \mathbf{w}$ were prepared via two steps from pyrazole carbaldehyde $\mathbf{5}$. Benefitting from the activated $\mathrm{C}-\mathrm{Cl}$ bond by the electron-withdrawing formyl group at the 4-position of the pyrazole ring, the introduction of either alkyl alcohol or substituted phenol nucleophiles into 5-chloro-1,3-dimethyl-1H-pyrazole-4-carbaldehyde (5) was performed smoothly and successfully, and afforded 5-substituted pyrazole-4-carbaldehydes $(\mathbf{6 a}-\mathbf{6 w})$. The condensation of intermediates 4 and $7 \mathbf{a}-7 \mathbf{w}$ proceeded smoothly with $\mathrm{Cs}_{2} \mathrm{CO}_{3}$ as the base and acetonitrile as the solvent to provide the title compounds $\mathbf{8 a}-\mathbf{8 w}$ in good yields. The structures of all the target compounds were analyzed and confirmed by ${ }^{1} \mathrm{H}-\mathrm{NMR},{ }^{13} \mathrm{C}-\mathrm{NMR}$ and elemental analyses. 
<smiles>CC(C)(C)C(=O)c1ccc(Oc2ccc(C(F)(F)F)cn2)cc1</smiles><smiles>[R]Oc1c(C=NOCc2ccc(Oc3ccc(C(F)(F)F)cn3)cc2)c(C)nn1C</smiles>

Scheme 1. Synthesis of the title compounds 8a-8w. Reagents and conditions: (a) 4-hydroxybenzaldehyde, $\mathrm{Cs}_{2} \mathrm{CO}_{3}, \mathrm{~N}, \mathrm{~N}$-dimethylformamide, $105{ }^{\circ} \mathrm{C}$ for $10 \mathrm{~h}, 75 \%$ for 2 ; (b) $\mathrm{NaBH}_{4}$, ethanol, $0{ }^{\circ} \mathrm{C}$ for $3 \mathrm{~h}, 90 \%$ for 3; (c) thionyl chloride, $N, N$-dimethylformamide, dichloromethane, r.t. for $8 \mathrm{~h}, 82 \%$ for 4 ; (d) $\mathrm{NaOR}$ $(\mathrm{R}=\mathrm{Me}$, Et and $t-\mathrm{Bu}), \mathrm{ROH}, 30{ }^{\circ} \mathrm{C}$ for $3 \mathrm{~h}, 45^{\circ} \mathrm{C}$ for $2 \mathrm{~h}, 41 \%-49 \%$ for $6 \mathrm{a}-6 \mathrm{c} ; \mathrm{NaOR}(\mathrm{R}=$ substituted phenyl), dimethylsulfoxide, $105{ }^{\circ} \mathrm{C}$ for $4-15 \mathrm{~h}, 60 \%-81 \%$ for $\mathbf{6 d}-\mathbf{6 w}$; (e) hydroxylamine hydrochloride, potassium hydroxide, methanol or ethanol, reflux for $6-16 \mathrm{~h}, 65 \%-87 \%$ for $7 \mathbf{a}-7 \mathbf{w}$; (f) compound 4 , $\mathrm{Cs}_{2} \mathrm{CO}_{3}$, acetonitrile, reflux for $10-18 \mathrm{~h}, 44 \%-63 \%$ for $8 \mathbf{a}-\mathbf{8 w}$.

\subsection{Biological Activities}

\subsubsection{Acaricidal Activity}

The acaricidal activity of all the title compounds against Tetranychus cinnabarinus was evaluated and the data are listed in Table 1 . The results indicated that 5-alkoxy pyrazole derivatives $\mathbf{8 a}, \mathbf{8 b}$ and $\mathbf{8 c}$ possessed no acaricidal activity at a concentration of $200 \mu \mathrm{g} / \mathrm{mL}$. For the other 5-aryloxy-substituted compounds $8 \mathbf{d}-\mathbf{8 w}$, an obvious substituent effect was found on the phenyl ring $C$. When the substituent at the 2-position of phenyl C (Figure 1) was halogen (8d, $\mathbf{8 g}$ and $\mathbf{8 j}$ ) or methoxy (8o), it would reduce the acaricidal activity, and the mortality obviously declined depending on the concentration and disappeared at the concentration of $10 \mu \mathrm{g} / \mathrm{mL}$. Moreover, 3-substituted phenyl C (Figure 1) affected the acaricidal activities in a similar manner. The introduction of halogen atoms ( $\mathbf{8} \mathbf{h}$ and $\mathbf{8 k}$ ) led to the loss of acaricidal activity, except for that of 3-fluoro derivative (8e). As well as unsubstituted compound $\mathbf{8 n}$, compound $\mathbf{8 e}$ retained $100.00 \%$ mortality, even at $10 \mu \mathrm{g} / \mathrm{mL}$. The results seem to show that compared with the inductive effect, steric hindrance of the 2- or 3-position on phenyl C (Figure 1) plays a more important role in regulating the acaricidal activity. In contrast, it exhibited a good tolerance of 4-substituents on phenyl C (Figure 1 and Table 1), because the introduction of halogen atoms (8f, $\mathbf{8 1}$ and $\mathbf{8 m})$, methoxy (8p), methyl (8q) or trifluoromethoxy (8s) did not affect the acaricidal activities at concentrations ranging from $200 \mu \mathrm{g} / \mathrm{mL}$ to $10 \mu \mathrm{g} / \mathrm{mL}$. However, 4-tert butyl compound $8 \mathbf{r}$ was an exception, whose activity against $T$. cinnabarinus disappeared completely at a concentration of $200 \mu \mathrm{g} / \mathrm{mL}$. Additionally, among disubstituted derivatives, compounds $8 \mathbf{t}$ and $\mathbf{8 w}$ displayed relatively higher acaricidal activity than compounds $8 \mathbf{u}$ and $\mathbf{8 v}$ from the concentrations of $200 \mu \mathrm{g} / \mathrm{mL}$ to $50 \mu \mathrm{g} / \mathrm{mL}$. 
Table 1. Acaricidal activities of compounds $\mathbf{8 a}-\mathbf{8 w}$ (mortality, \%).

\begin{tabular}{|c|c|c|c|c|c|}
\hline \multirow{2}{*}{ Compd. } & \multirow{2}{*}{$\mathbf{R}$} & \multicolumn{4}{|c|}{ Tetranychus cinnabarinus } \\
\hline & & $200 \mu \mathrm{g} / \mathrm{mL}$ & $100 \mu \mathrm{g} / \mathrm{mL}$ & $50 \mu \mathrm{g} / \mathrm{mL}$ & $10 \mu \mathrm{g} / \mathrm{mL}$ \\
\hline $8 a$ & $\mathrm{Me}$ & 0 & $-\mathrm{b}$ & - & - \\
\hline $8 b$ & Et & 0 & - & - & - \\
\hline $8 c$ & $t-\mathrm{Bu}$ & 0 & - & - & - \\
\hline $8 d$ & $2-\mathrm{FC}_{6} \mathrm{H}_{4}$ & $100.00 \pm 0.00$ & $90.32 \pm 1.38$ & $70.43 \pm 0.82$ & 0 \\
\hline $8 \mathrm{e}$ & $3-\mathrm{FC}_{6} \mathrm{H}_{4}$ & $100.00 \pm 0.00$ & $100.00 \pm 0.00$ & $100.00 \pm 0.00$ & $100.00 \pm 0.00$ \\
\hline $8 f$ & $4-\mathrm{FC}_{6} \mathrm{H}_{4}$ & $100.00 \pm 0.00$ & $100.00 \pm 0.00$ & $100.00 \pm 0.00$ & $100.00 \pm 0.00$ \\
\hline $8 g$ & $2-\mathrm{ClC}_{6} \mathrm{H}_{4}$ & $70.28 \pm 1.33^{a}$ & 0 & - & - \\
\hline $8 \mathrm{~h}$ & $3-\mathrm{ClC}_{6} \mathrm{H}_{4}$ & $100.00 \pm 0.00$ & $30.39 \pm 0.67$ & - & - \\
\hline $8 \mathrm{i}$ & $4-\mathrm{ClC}_{6} \mathrm{H}_{4}$ & $100.00 \pm 0.00$ & $100.00 \pm 0.00$ & $100.00 \pm 0.00$ & $50.49 \pm 1.78$ \\
\hline $8 \mathbf{j}$ & $2-\mathrm{BrC}_{6} \mathrm{H}_{4}$ & $80.16 \pm 0.38$ & $30.46 \pm 1.52$ & 0 & - \\
\hline $8 k$ & $3-\mathrm{BrC}_{6} \mathrm{H}_{4}$ & $70.33 \pm 1.22$ & 0 & - & - \\
\hline 81 & $4-\mathrm{BrC}_{6} \mathrm{H}_{4}$ & $100.00 \pm 0.00$ & $100.00 \pm 0.00$ & $100.00 \pm 0.00$ & $100.00 \pm 0.00$ \\
\hline $8 m$ & $4-\mathrm{IC}_{6} \mathrm{H}_{4}$ & $100.00 \pm 0.00$ & $100.00 \pm 0.00$ & $100.00 \pm 0.00$ & $100.00 \pm 0.00$ \\
\hline $8 n$ & $\mathrm{C}_{6} \mathrm{H}_{5}$ & $100.00 \pm 0.00$ & $100.00 \pm 0.00$ & $100.00 \pm 0.00$ & $100.00 \pm 0.00$ \\
\hline 80 & $2-\mathrm{OMeC}_{6} \mathrm{H}_{4}$ & 0 & - & - & - \\
\hline $8 p$ & $4-\mathrm{OMeC}_{6} \mathrm{H}_{4}$ & $100.00 \pm 0.00$ & $100.00 \pm 0.00$ & $100.00 \pm 0.00$ & $100.00 \pm 0.00$ \\
\hline $8 q$ & $4-\mathrm{MeC}_{6} \mathrm{H}_{4}$ & $100.00 \pm 0.00$ & $100.00 \pm 0.00$ & $100.00 \pm 0.00$ & $100.00 \pm 0.00$ \\
\hline $8 r$ & $4-t-\mathrm{BuC}_{6} \mathrm{H}_{4}$ & 0 & - & - & - \\
\hline $8 s$ & $4-\mathrm{OCF}_{3} \mathrm{C}_{6} \mathrm{H}_{4}$ & $100.00 \pm 0.00$ & $100.00 \pm 0.00$ & $100.00 \pm 0.00$ & $95.29 \pm 1.45$ \\
\hline $8 t$ & $2,3-\mathrm{F}_{2} \mathrm{C}_{6} \mathrm{H}_{3}$ & $100.00 \pm 0.00$ & $100.00 \pm 0.00$ & $80.42 \pm 1.56$ & - \\
\hline $8 u$ & $2,4-\mathrm{Cl}_{2} \mathrm{C}_{6} \mathrm{H}_{3}$ & $80.17 \pm 0.82$ & $50.23 \pm 1.43$ & 0 & - \\
\hline $8 v$ & $2,3-\mathrm{Me}_{2} \mathrm{C}_{6} \mathrm{H}_{3}$ & 0 & - & - & - \\
\hline $8 w$ & $2,4-\mathrm{Me}_{2} \mathrm{C}_{6} \mathrm{H}_{3}$ & $100.00 \pm 0.00$ & $100.00 \pm 0.00$ & $60.35 \pm 1.45$ & - \\
\hline Fenpyroximate & & $100.00 \pm 0.00$ & $100.00 \pm 0.00$ & $100.00 \pm 0.00$ & $100.00 \pm 0.00$ \\
\hline
\end{tabular}

a Each value represents the mean \pm standard error of three replications; ${ }^{\mathrm{b}}$ "- - " refers to "not tested".

\subsubsection{Insecticidal Activities}

Besides acaricidal potencies, the insecticidal activities of the new compounds were also explored on Plutella xylostella and Aphis craccivora. As shown in Table 2, some of the obtained compounds displayed good insecticidal activities against $P$. xylostella. For instance, the mortalities of compounds 8e, 8f, 8i, 81, 8m, 8n, 8p, and 8q against P. xylostella were all $100.00 \%$ at $200 \mu \mathrm{g} / \mathrm{mL}$. Moreover, some of them showed good insecticidal activities against $P$. xylostella when the concentration was reduced to $50 \mu \mathrm{g} / \mathrm{mL}$; compounds $\mathbf{8 e}$ and $\mathbf{8 1}$ possessed relatively higher insecticidal activities against P. xylostella than other derivatives. Some target compounds also demonstrated moderate to good insecticidal activities against $A$. craccivora at $200 \mu \mathrm{g} / \mathrm{mL}$; for example, compounds $\mathbf{8 e}, \mathbf{8 h}, \mathbf{8 i}$, and $\mathbf{8 r}$ had $100.00 \%, 85.33 \%, 95.28 \%$, and $100.00 \%$ inhibition rates, respectively. In fact, similar to that of acaricidal activities, the compounds possessing more potent insecticidal abilities against $P$. xylostella were 4-substituted derivatives with compound $\mathbf{8 e}$ as the only exception. When it comes to A. craccivora, the structure-activity relationships were not obvious. Overall, they were more potent against $P$. xylostella than against $A$. craccivora.

All the above data implied that structural modification of Fenpyroximate by a 5-trifluoromethyl pyridyl unit could produce some new compounds with good biological activities. To get more active derivatives, further analogue synthesis and structural optimization are well under way. 
Table 2. Insecticidal activities of compounds $\mathbf{8 a}-\mathbf{8 w}$ (mortality, \%).

\begin{tabular}{|c|c|c|c|c|c|}
\hline \multirow{2}{*}{ Compd. } & \multirow{2}{*}{$\mathbf{R}$} & \multicolumn{2}{|c|}{ Plutella xylostella } & \multicolumn{2}{|c|}{ Aphis craccivora } \\
\hline & & $200 \mu \mathrm{g} / \mathrm{mL}$ & $50 \mu \mathrm{g} / \mathrm{mL}$ & $200 \mu \mathrm{g} / \mathrm{mL}$ & $100 \mu \mathrm{g} / \mathrm{mL}$ \\
\hline $8 a$ & $\mathrm{Me}$ & $100.00 \pm 0.00$ & 0 & $30.26 \pm 1.56$ & - \\
\hline $8 b$ & Et & 0 & $-{ }^{b}$ & 0 & - \\
\hline $8 c$ & $t-\mathrm{Bu}$ & 0 & - & 0 & - \\
\hline $8 d$ & $2-\mathrm{FC}_{6} \mathrm{H}_{4}$ & 0 & - & 0 & - \\
\hline $8 e$ & $3-\mathrm{FC}_{6} \mathrm{H}_{4}$ & $100.00 \pm 0.00$ & $86.42 \pm 0.88$ & $100.00 \pm 0.00$ & $60.18 \pm 1.33$ \\
\hline $8 f$ & $4-\mathrm{FC}_{6} \mathrm{H}_{4}$ & $100.00 \pm 0.00$ & $43.36 \pm 1.07$ & $60.29 \pm 1.52$ & $30.41 \pm 0.71$ \\
\hline $8 g$ & $2-\mathrm{ClC}_{6} \mathrm{H}_{4}$ & 0 & - & 0 & - \\
\hline $8 \mathrm{~h}$ & $3-\mathrm{ClC}_{6} \mathrm{H}_{4}$ & $30.29 \pm 1.55^{\mathrm{a}}$ & 0 & $85.33 \pm 1.25$ & $50.27 \pm 1.06$ \\
\hline $8 \mathrm{i}$ & $4-\mathrm{ClC}_{6} \mathrm{H}_{4}$ & $100.00 \pm 0.00$ & $71.23 \pm 0.95$ & $95.28 \pm 1.32$ & $60.26 \pm 1.69$ \\
\hline $8 \mathbf{j}$ & $2-\mathrm{BrC}_{6} \mathrm{H}_{4}$ & 0 & - & 20.41 & - \\
\hline $8 k$ & $3-\mathrm{BrC}_{6} \mathrm{H}_{4}$ & $57.35 \pm 0.54$ & $29.16 \pm 1.86$ & 0 & - \\
\hline 81 & $4-\mathrm{BrC}_{6} \mathrm{H}_{4}$ & $100.00 \pm 0.00$ & $100.00 \pm 0.00$ & $50.37 \pm 1.32$ & 0 \\
\hline $8 m$ & $4-\mathrm{IC}_{6} \mathrm{H}_{4}$ & $100.00 \pm 0.00$ & $71.39 \pm 1.53$ & 0 & - \\
\hline $8 n$ & $\mathrm{C}_{6} \mathrm{H}_{5}$ & $100.00 \pm 0.00$ & $71.18 \pm 0.65$ & $70.25 \pm 1.63$ & $50.43 \pm 1.38$ \\
\hline 80 & $2-\mathrm{OMeC}_{6} \mathrm{H}_{4}$ & 0 & - & 0 & - \\
\hline $8 p$ & $4-\mathrm{OMeC}_{6} \mathrm{H}_{4}$ & $100.00 \pm 0.00$ & $43.41 \pm 1.71$ & $70.32 \pm 1.95$ & $20.17 \pm 0.71$ \\
\hline $8 q$ & $4-\mathrm{MeC}_{6} \mathrm{H}_{4}$ & $100.00 \pm 0.00$ & $71.22 \pm 1.24$ & 0 & - \\
\hline $8 r$ & $4-t-\mathrm{BuC}_{6} \mathrm{H}_{4}$ & 0 & - & $100.00 \pm 0.00$ & $40.09 \pm 0.36$ \\
\hline $8 s$ & $4-\mathrm{OCF}_{3} \mathrm{C}_{6} \mathrm{H}_{4}$ & $57.27 \pm 0.83$ & $43.35 \pm 1.65$ & - & - \\
\hline $8 t$ & $2,3-\mathrm{F}_{2} \mathrm{C}_{6} \mathrm{H}_{3}$ & $43.09 \pm 1.26$ & 0 & - & - \\
\hline $8 u$ & $2,4-\mathrm{Cl}_{2} \mathrm{C}_{6} \mathrm{H}_{3}$ & $43.25 \pm 1.07$ & 0 & - & - \\
\hline $8 v$ & $2,3-\mathrm{Me}_{2} \mathrm{C}_{6} \mathrm{H}_{3}$ & 0 & - & - & - \\
\hline $8 w$ & $2,4-\mathrm{Me}_{2} \mathrm{C}_{6} \mathrm{H}_{3}$ & $43.18 \pm 0.92$ & 0 & - & - \\
\hline Pyridalyl & & $100.00 \pm 0.00$ & $100.00 \pm 0.00$ & - & - \\
\hline Imidacloprid & & - & - & $100.00 \pm 0.00$ & $100.00 \pm 0.00$ \\
\hline
\end{tabular}

a Each value represents the mean \pm standard error of three replications; " "- " refers to "not tested".

\section{Experimental Section}

\subsection{Chemistry}

\subsubsection{General Procedures}

All reagents were chemically pure and solvents were dried according to standard methods. The ${ }^{1} \mathrm{H}-\mathrm{NMR}$ and ${ }^{13} \mathrm{C}-\mathrm{NMR}$ spectra were obtained on a Bruker AV400 spectrometer $\left(400 \mathrm{MHz},{ }^{1} \mathrm{H}\right.$; $100 \mathrm{MHz},{ }^{13} \mathrm{C}$, Bruker, Billerica, MA, USA) in $\mathrm{CDCl}_{3}$ with tetramethylsilane as the internal standard. The melting points were determined on an X-4 binocular microscope melting point apparatus (Beijing Tech Instrument Co., Beijing, China) and are uncorrected. Elemental analyses were determined on a Yanaco CHN Corder MT-3 elemental analyzer (Yanaco, Kyoto, Japan). The reactions were monitored by analytical thin-layer chromatography (TLC) with ultraviolet (UV) light and TLC was carried out on silica gel $\mathrm{GF}_{254}$. The intermediates 5-chloropyrazole aldehyde 5 and 5-alkoxy pyrazole aldehyde $\mathbf{6 a - 6 c}$ were synthesized according to the reported procedures [26]. The 5-Substituted pyrazole oximes $7 \mathbf{a}-7 \mathbf{w}$ were prepared by the literature method [11].

\subsubsection{Synthesis of 4-(5-Trifluoromethylpyridin-2-yloxy)benzaldehyde (2)}

To a solution of 4-hydroxybenzaldehyde $(6.4 \mathrm{~g}, 52.5 \mathrm{mmol})$ in $\mathrm{N}, \mathrm{N}$-dimethylformamide $(150 \mathrm{~mL})$ was added $\mathrm{Cs}_{2} \mathrm{CO}_{3}(16.3 \mathrm{~g}$, $50 \mathrm{mmol})$, the mixture was then stirred for $20 \mathrm{~min}$ at room temperature, followed by adding compound $1(9.1 \mathrm{~g}, 50 \mathrm{mmol})$. The resulting mixture was then heated slowly to $105^{\circ} \mathrm{C}$ and stirred for $10 \mathrm{~h}$. After cooled to room temperature, the solvent was evaporated in vacuo. The slurry was then distributed in water $(150 \mathrm{~mL})$ and ethyl acetate $(100 \mathrm{~mL})$, and the separated water phase was then extracted with ethyl acetate $(3 \times 50 \mathrm{~mL})$. The combined organic layer was washed by water and brine, dried over anhydrous $\mathrm{Na}_{2} \mathrm{SO}_{4}$, and concentrated in rotatory evaporator to afford 
compound 2 in 75\% yield as a white solid, which was used for the following transformations without further purification.

\subsubsection{Synthesis of 4-(5-Trifluoromethylpyridin-2-yloxy)phenylmethanol (3)}

Intermediate $2(13.4 \mathrm{~g}, 50 \mathrm{mmol})$ was dissolved in ethanol $(100 \mathrm{~mL})$ and cooled to $0{ }^{\circ} \mathrm{C}$. To the solution was added $\mathrm{NaBH}_{4}(3.8 \mathrm{~g}, 100 \mathrm{mmol})$ in portions over $30 \mathrm{~min}$. After being stirred at $0{ }^{\circ} \mathrm{C}$ for $3 \mathrm{~h}$, the reaction mixture was poured into water, followed by adding $5 \%$ hydrochloric acid to adjust $\mathrm{pH}$ to 5-6. The resulting solution was extracted by chloroform $(3 \times 50 \mathrm{~mL})$, dried over anhydrous $\mathrm{Na}_{2} \mathrm{SO}_{4}$ and concentrated under reduced pressure to give compound 3 in $90 \%$ yield, which was used in the next procedure without further purification.

\subsubsection{Synthesis of 2-(4-Chloromethylphenoxy)-5-(trifluoromethyl)pyridine (4)}

A solution of intermediate $3(13.5 \mathrm{~g}, 50 \mathrm{mmol})$ in dichloromethane $(80 \mathrm{~mL})$ was cooled in ice-water bath followed by adding thionyl chloride $(8.9 \mathrm{~g}, 74.8 \mathrm{mmol})$ dropwise over $20 \mathrm{~min}$. Then a few drops of $\mathrm{N}, \mathrm{N}$-dimethylformamide was added thereto. The resulting solution was stirred at room temperature for $8 \mathrm{~h}$. The reaction mixture was quenched by trash ice, then the organic phase was separated, washed by water and saturated $\mathrm{NaHCO}_{3}$, dried over anhydrous $\mathrm{Na}_{2} \mathrm{SO}_{4}$, and evaporated in vacuo to produce compound 4 (yield 82\%) as a white solid; ${ }^{1} \mathrm{H}-\mathrm{NMR}\left(\mathrm{CDCl}_{3}\right): \delta 8.36(\mathrm{~s}, 1 \mathrm{H}, \mathrm{Py}-\mathrm{H}), 7.84\left(\mathrm{dd}, J_{1}=8.8 \mathrm{~Hz}\right.$, $\left.J_{2}=2.4 \mathrm{~Hz}, 1 \mathrm{H}, \mathrm{Py}-\mathrm{H}\right), 7.38(\mathrm{~d}, J=8.4 \mathrm{~Hz}, 2 \mathrm{H}, \mathrm{Ar}-\mathrm{H}), 7.07(\mathrm{~d}, J=8.4 \mathrm{~Hz}, 2 \mathrm{H}, \mathrm{Ar}-\mathrm{H}), 6.96(\mathrm{~d}, J=8.8 \mathrm{~Hz}$, $1 \mathrm{H}, \mathrm{Py}-\mathrm{H}), 4.54\left(\mathrm{~s}, 2 \mathrm{H}, \mathrm{CH}_{2}\right)$. Anal. Calcd for $\mathrm{C}_{13} \mathrm{H}_{9} \mathrm{ClF}_{3} \mathrm{NO}$ : C 54.28; H 3.15; N 4.87. Found: C 54.41; H $3.03 ; \mathrm{N} 4.70$.

\subsubsection{General Procedure for the Preparation of $6 \mathrm{~d}-6 \mathrm{w}$}

To a solution of substituted phenol $(26 \mathrm{mmol})$ in absolute ethanol $(50 \mathrm{~mL})$ was added sodium hydroxide $(26 \mathrm{mmol})$ at room temperature. The mixture was heated to reflux for 3-5 h. After the removal of the solvent, the residue was dissolved in dimethylsulfoxide $(50 \mathrm{~mL})$, to the resulting mixture was added 5-chloro-1,3-dimethyl-1H-pyrazole-4-carbaldehyde (5) (20 mmol) in portions. Then the solution was heated to $105^{\circ} \mathrm{C}$ and maintained at that temperature for $4-15 \mathrm{~h}$ and cooled to room temperature. The reaction mixture was poured into water $(100 \mathrm{~mL})$ and extracted with ethyl acetate $(3 \times 50 \mathrm{~mL})$. The organic layer was washed with water $(3 \times 25 \mathrm{~mL})$ and dried over anhydrous $\mathrm{Na}_{2} \mathrm{SO}_{4}$, filtered and evaporated to produce the corresponding carbaldehydes $\mathbf{6} \mathbf{d}-\mathbf{6} \mathbf{w}$, with yields ranging from $60 \%$ to $81 \%$ [11].

\subsubsection{General Procedure for the Preparation of $\mathbf{8 a - 8 w}$}

To a stirred solution of intermediate $4(7.2 \mathrm{mmol})$, compound $7(6 \mathrm{mmol})$ in anhydrous acetonitrile $(30 \mathrm{~mL})$ was added $\mathrm{Cs}_{2} \mathrm{CO}_{3}(7.2 \mathrm{mmol})$ at room temperature, the resulting mixture was heated to reflux for 10-18 h. After cooled to room temperature, the reaction mixture was filtered. After most of the solvent had been evaporated under reduced pressure, the residue was admixed with water $(100 \mathrm{~mL})$ and extracted with dichloromethane $(3 \times 30 \mathrm{~mL})$. The combined organic layer was washed with water $(3 \times 30 \mathrm{~mL})$, and dried over anhydrous $\mathrm{Na}_{2} \mathrm{SO}_{4}$. The solvent was removed using a rotary evaporator to give a residue, which was then separated by silica gel column chromatography using petroleum ether and ethyl acetate $(v / v=30: 1)$ as eluent to afford the target compounds $\mathbf{8 a}-\mathbf{8 w}$, with yields ranging from $44 \%$ to $63 \%$. All 23 pyrazole oxime derivatives 8a-8w were novel and the physical and spectral data for these compounds are listed below.

1,3-Dimethyl-5-methyloxy-1H-pyrazole-4-carbaldehyde-O-[4-(5-trifluoromethylpyridin-2-yloxy)phenylmethyl]oxime (8a): White oil, yield 52\%. ${ }^{1} \mathrm{H}-\mathrm{NMR}\left(\mathrm{CDCl}_{3}\right): \delta 8.44(\mathrm{~s}, 1 \mathrm{H}, \mathrm{Py}-\mathrm{H}), 8.08(\mathrm{~s}, 1 \mathrm{H}, \mathrm{CH}=\mathrm{N}), 7.90(\mathrm{~d}$, $J=8.8 \mathrm{~Hz}, 1 \mathrm{H}, \mathrm{Py}-\mathrm{H}), 7.49(\mathrm{~d}, J=7.6 \mathrm{~Hz}, 2 \mathrm{H}, \mathrm{Ar}-\mathrm{H}$ and Py-H), $7.15(\mathrm{~d}, J=7.2 \mathrm{~Hz}, 2 \mathrm{H}, \mathrm{Ar}-\mathrm{H}), 7.01(\mathrm{~d}$, $J=8.8 \mathrm{~Hz}, 1 \mathrm{H}, \mathrm{Ar}-\mathrm{H}), 5.16\left(\mathrm{~s}, 2 \mathrm{H}, \mathrm{CH}_{2}\right), 3.94\left(\mathrm{~s}, 3 \mathrm{H}, \mathrm{OCH}_{3}\right), 3.62\left(\mathrm{~s}, 3 \mathrm{H}, \mathrm{N}-\mathrm{CH}_{3}\right), 2.28\left(\mathrm{~s}, 3 \mathrm{H}, \mathrm{CH}_{3}\right)$; ${ }^{13} \mathrm{C}-\mathrm{NMR}\left(\mathrm{CDCl}_{3}\right): \delta 165.8,153.0,152.8,146.8,145.5,145.4,141.6,136.7,136.6,135.2,130.0,121.4,111.3$, 
97.4, 75.4, 61.7, 33.6, 14.0. Anal. Calcd for $\mathrm{C}_{20} \mathrm{H}_{19} \mathrm{~F}_{3} \mathrm{~N}_{4} \mathrm{O}_{3}$ : C 57.14; $\mathrm{H}$ 4.56; $\mathrm{N}$ 13.33. Found: C 57.28; H 4.39; N 13.16 .

1,3-Dimethyl-5-ethyloxy-1H-pyrazole-4-carbaldehyde-O-[4-(5-trifluoromethylpyridin-2-yloxy)phenylmethyl]oxime (8b). White solid, yield 55\%, mp $48-50{ }^{\circ} \mathrm{C} .{ }^{1} \mathrm{H}-\mathrm{NMR}\left(\mathrm{CDCl}_{3}\right): \delta 8.42(\mathrm{~s}, 1 \mathrm{H}, \mathrm{Py}-\mathrm{H}), 8.04(\mathrm{~s}, 1 \mathrm{H}$, $\mathrm{CH}=\mathrm{N}), 7.88(\mathrm{~d}, J=8.8 \mathrm{~Hz}, 1 \mathrm{H}, \mathrm{Py}-\mathrm{H}), 7.47(\mathrm{~d}, J=7.2 \mathrm{~Hz}, 2 \mathrm{H}, \mathrm{Ar}-\mathrm{H}$ and Py-H), $7.14(\mathrm{~d}, J=6.8 \mathrm{~Hz}$, 2H, Ar-H), $6.99(\mathrm{~d}, J=8.4 \mathrm{~Hz}, 1 \mathrm{H}, \mathrm{Ar}-\mathrm{H}), 5.14\left(\mathrm{~s}, 2 \mathrm{H}, \mathrm{CH}_{2}\right), 4.16\left(\mathrm{q}, J=6.8 \mathrm{~Hz}, 2 \mathrm{H}, \mathrm{CH}_{2}\right), 3.60(\mathrm{~s}, 3 \mathrm{H}$, $\left.\mathrm{N}-\mathrm{CH}_{3}\right), 2.26\left(\mathrm{~s}, 3 \mathrm{H}, \mathrm{CH}_{3}\right), 1.32\left(\mathrm{t}, J=6.0 \mathrm{~Hz}, 3 \mathrm{H}, \mathrm{CH}_{3}\right) ;{ }^{13} \mathrm{C}-\mathrm{NMR}\left(\mathrm{CDCl}_{3}\right): \delta 165.8,152.8,152.1,146.8$, 145.4, 145.3, 141.7, 136.7, 136.6, 135.3, 130.0, 121.4, 111.3, 97.8, 75.3, 70.6, 33.6, 15.3, 14.0. Anal. Calcd for $\mathrm{C}_{21} \mathrm{H}_{21} \mathrm{~F}_{3} \mathrm{~N}_{4} \mathrm{O}_{3}$ : C 58.06; $\mathrm{H} 4.87 ; \mathrm{N}$ 12.90. Found: C 58.23; H 4.69; N 12.76.

1,3-Dimethyl-5-tert-butyloxy-1H-pyrazole-4-carbaldehyde-O-[4-(5-trifluoromethylpyridin-2-yloxy)phenylmethyl]oxime (8c). White solid, yield 50\%, mp 64-65 ${ }^{\circ} \mathrm{C} .{ }^{1} \mathrm{H}-\mathrm{NMR}\left(\mathrm{CDCl}_{3}\right): \delta 8.45(\mathrm{~s}, 1 \mathrm{H}, \mathrm{Py}-\mathrm{H}), 8.01(\mathrm{~s}, 1 \mathrm{H}$, $\mathrm{CH}=\mathrm{N}), 7.90(\mathrm{~d}, J=8.4 \mathrm{~Hz}, 1 \mathrm{H}, \mathrm{Py}-\mathrm{H}), 7.49(\mathrm{~d}, J=8.0 \mathrm{~Hz}, 2 \mathrm{H}, \mathrm{Ar}-\mathrm{H}$ and Py-H), $7.15(\mathrm{~d}, J=7.2 \mathrm{~Hz}, 2 \mathrm{H}$, Ar-H), $7.01(\mathrm{~d}, J=8.8 \mathrm{~Hz}, 1 \mathrm{H}, \mathrm{Ar}-\mathrm{H}), 5.15\left(\mathrm{~s}, 2 \mathrm{H}, \mathrm{CH}_{2}\right), 3.62\left(\mathrm{~s}, 3 \mathrm{H}, \mathrm{N}-\mathrm{CH}_{3}\right), 2.35\left(\mathrm{~s}, 3 \mathrm{H}, \mathrm{CH}_{3}\right), 1.38(\mathrm{~s}$, $\left.9 \mathrm{H}, t-\mathrm{C}_{4} \mathrm{H}_{9}\right) ;{ }^{13} \mathrm{C}-\mathrm{NMR}\left(\mathrm{CDCl}_{3}\right): \delta 165.8,152.7,150.1,146.6,145.5,145.4,143.0,136.7,135.3,130.0,125.1$, 121.3, 111.3, 101.6, 85.4, 75.3, 34.6, 29.0, 15.2. Anal. Calcd for $\mathrm{C}_{23} \mathrm{H}_{25} \mathrm{~F}_{3} \mathrm{~N}_{4} \mathrm{O}_{3}: \mathrm{C} 59.73 ; \mathrm{H} 5.45 ; \mathrm{N} 12.11$. Found: C 59.62; H 5.58; N 12.30 .

1,3-Dimethyl-5-(2-fluorophenoxy)-1H-pyrazole-4-carbaldehyde-O-[4-(5-trifluoromethylpyridin-2-yloxy)phenylmethyl]oxime (8d). Yellow solid, yield 48\%, mp 56-58 ${ }^{\circ} \mathrm{C} .{ }^{1} \mathrm{H}-\mathrm{NMR}\left(\mathrm{CDCl}_{3}\right): \delta 8.46(\mathrm{~s}, 1 \mathrm{H}, \mathrm{Py}-\mathrm{H}), 7.91(\mathrm{~d}$, $J=8.4 \mathrm{~Hz}, 1 \mathrm{H}, \mathrm{Py}-\mathrm{H}), 7.84(\mathrm{~s}, 1 \mathrm{H}, \mathrm{CH}=\mathrm{N}), 7.37(\mathrm{~d}, J=6.8 \mathrm{~Hz}, 2 \mathrm{H}, \mathrm{Ar}-\mathrm{H}$ and $\mathrm{Py}-\mathrm{H}), 7.01-7.22(\mathrm{~m}, 6 \mathrm{H}$, ArH), 6.78-6.82 (m, 1H, ArH), $5.01\left(\mathrm{~s}, 2 \mathrm{H}, \mathrm{CH}_{2}\right), 3.68\left(\mathrm{~s}, 3 \mathrm{H}, \mathrm{N}-\mathrm{CH}_{3}\right), 2.38\left(\mathrm{~s}, 3 \mathrm{H}, \mathrm{CH}_{3}\right) .{ }^{13} \mathrm{C}-\mathrm{NMR}$ $\left(\mathrm{CDCl}_{3}\right): \delta 165.8,153.2,152.8,150.8,147.3,147.0,145.5,145.4,144.3,144.2,140.4,136.7,135.0,130.0$, 124.6, 124.5, 121.3, 117.2, 117.1, 116.8, 111.3, 99.9, 75.5, 34.2, 14.5. Anal. Calcd for $\mathrm{C}_{25} \mathrm{H}_{20} \mathrm{~F}_{4} \mathrm{~N}_{4} \mathrm{O}_{3}$ : C 60.00; H 4.03; N 11.20. Found: C 60.19; H 3.85; N 11.02.

1,3-Dimethyl-5-(3-fluorophenoxy)-1H-pyrazole-4-carbaldehyde-O-[4-(5-trifluoromethylpyridin-2-yloxy)phenylmethyl] -oxime (8e). White oil, yield 51\%. ${ }^{1} \mathrm{H}-\mathrm{NMR}\left(\mathrm{CDCl}_{3}\right): \delta 8.45(\mathrm{~s}, 1 \mathrm{H}, \mathrm{Py}-\mathrm{H}), 7.91(\mathrm{~d}, J=8.8 \mathrm{~Hz}, 1 \mathrm{H}, \mathrm{Py}-\mathrm{H})$, $7.86(\mathrm{~s}, 1 \mathrm{H}, \mathrm{CH}=\mathrm{N}), 7.37(\mathrm{~d}, J=7.2 \mathrm{~Hz}, 2 \mathrm{H}, \mathrm{Ar}-\mathrm{H}$ and Py-H), 7.25-7.31 (m, 1H, ArH), $7.11(\mathrm{~d}, J=6.8 \mathrm{~Hz}$, $2 \mathrm{H}, \mathrm{Ar}-\mathrm{H}), 7.01(\mathrm{~d}, J=8.4 \mathrm{~Hz}, 1 \mathrm{H}, \mathrm{Ar}-\mathrm{H}), 6.65-6.85(\mathrm{~m}, 3 \mathrm{H}, \mathrm{ArH}), 5.03\left(\mathrm{~s}, 2 \mathrm{H}, \mathrm{CH}_{2}\right), 3.63\left(\mathrm{~s}, 3 \mathrm{H}, \mathrm{N}-\mathrm{CH}_{3}\right)$, $2.40\left(\mathrm{~s}, 3 \mathrm{H}, \mathrm{CH}_{3}\right) .{ }^{13} \mathrm{C}-\mathrm{NMR}\left(\mathrm{CDCl}_{3}\right): \delta 165.8,164.8,162.3,157.6,152.8,147.0,145.5,140.5,136.7,134.9$, 130.9, 130.8, 130.0, 125.1, 121.3, 111.3, 110.9, 110.8, 110.6, 103.7, 103.4, 100.4, 75.5, 34.2, 14.6. Anal. Calcd for $\mathrm{C}_{25} \mathrm{H}_{20} \mathrm{~F}_{4} \mathrm{~N}_{4} \mathrm{O}_{3}$ : C 60.00; H 4.03; N 11.20. Found: C 59.85; H 4.21; N 11.39.

1,3-Dimethyl-5-(4-fluorophenoxy)-1H-pyrazole-4-carbaldehyde-O-[4-(5-trifluoromethylpyridin-2-yloxy)phenylmethyl] -oxime (8f). White solid, yield 56\%, mp 46-48 ${ }^{\circ} \mathrm{C} .{ }^{1} \mathrm{H}-\mathrm{NMR}\left(\mathrm{CDCl}_{3}\right): \delta 8.44(\mathrm{~s}, 1 \mathrm{H}, \mathrm{Py}-\mathrm{H}), 7.90(\mathrm{~d}$, $J=8.4 \mathrm{~Hz}, 1 \mathrm{H}, \mathrm{Py}-\mathrm{H}), 7.83(\mathrm{~s}, 1 \mathrm{H}, \mathrm{CH}=\mathrm{N}), 7.36(\mathrm{~d}, J=8.0 \mathrm{~Hz}, 2 \mathrm{H}, \mathrm{Ar}-\mathrm{H}$ and Py-H), $7.11(\mathrm{~d}, J=8.4 \mathrm{~Hz}$, 2H, Ar-H), 6.98-7.02 (m, 3H, ArH), 6.86-6,88 (m, 2H, ArH), $5.02\left(\mathrm{~s}, 2 \mathrm{H}, \mathrm{CH}_{2}\right), 3.62\left(\mathrm{~s}, 3 \mathrm{H}, \mathrm{N}_{-} \mathrm{CH}_{3}\right)$, $2.38\left(\mathrm{~s}, 3 \mathrm{H}, \mathrm{CH}_{3}\right) .{ }^{13} \mathrm{C}-\mathrm{NMR}\left(\mathrm{CDCl}_{3}\right): \delta 165.8,160.0,157.5,152.8,152.7,147.7,147.0,145.5,145.4,140.6$, 136.7, 135.0, 130.0, 125.1, 121.3, 120.0, 116.6, 116.5, 116.4, 111.4, 100.1, 75.4, 34.2, 14.6. Anal. Calcd for $\mathrm{C}_{25} \mathrm{H}_{20} \mathrm{~F}_{4} \mathrm{~N}_{4} \mathrm{O}_{3}$ : C 60.00; H 4.03; N 11.20. Found: C 60.17; H 3.89; N 11.08.

1,3-Dimethyl-5-(2-chlorophenoxy)-1H-pyrazole-4-carbaldehyde-O-[4-(5-trifluoromethylpyridin-2-yloxy)phenylmethyl] -oxime (8g). White solid, yield 53\%, mp 46-48 ${ }^{\circ} \mathrm{C} .{ }^{1} \mathrm{H}-\mathrm{NMR}\left(\mathrm{CDCl}_{3}\right): \delta 8.46(\mathrm{~s}, 1 \mathrm{H}, \mathrm{Py}-\mathrm{H}), 7.91(\mathrm{~d}$, $J=8.8 \mathrm{~Hz}, 1 \mathrm{H}, \mathrm{Py}-\mathrm{H}), 7.82(\mathrm{~s}, 1 \mathrm{H}, \mathrm{CH}=\mathrm{N}), 7.46(\mathrm{~d}, J=7.6 \mathrm{~Hz}, 1 \mathrm{H}, \mathrm{Ar}-\mathrm{H}), 7.36(\mathrm{~d}, J=7.6 \mathrm{~Hz}, 2 \mathrm{H}, \mathrm{Ar}-\mathrm{H}$ and Py-H), 7.05-7.19 (m, 4H, ArH), $7.01(\mathrm{~d}, J=8.8 \mathrm{~Hz}, 1 \mathrm{H}, \mathrm{Ar}-\mathrm{H}), 6.71(\mathrm{~d}, J=8.0 \mathrm{~Hz}, 1 \mathrm{H}, \mathrm{Ar}-\mathrm{H}), 5.01(\mathrm{~s}$, $\left.2 \mathrm{H}, \mathrm{CH}_{2}\right), 3.66\left(\mathrm{~s}, 3 \mathrm{H}, \mathrm{N}-\mathrm{CH}_{3}\right), 2.38\left(\mathrm{~s}, 3 \mathrm{H}, \mathrm{CH}_{3}\right) .{ }^{13} \mathrm{C}-\mathrm{NMR}\left(\mathrm{CDCl}_{3}\right): \delta 165.8,152.8,152.2,147.1,147.0$, 145.6, 145.5, 145.4, 140.3, 136.7, 134.9, 131.0, 130.1, 128.0, 124.6, 122.8, 121.3, 115.6, 111.3, 100.2, 75.5, 34.2, 14.5. Anal. Calcd for $\mathrm{C}_{25} \mathrm{H}_{20} \mathrm{ClF}_{3} \mathrm{~N}_{4} \mathrm{O}_{3}$ : C 58.09; H 3.90; N 10.84. Found: C 58.22; H 3.78; N 10.65.

1,3-Dimethyl-5-(3-chlorophenoxy)-1H-pyrazole-4-carbaldehyde-O-[4-(5-trifluoromethylpyridin-2-yloxy)phenylmethyl] -oxime (8h). White oil, yield 55\%. ${ }^{1} \mathrm{H}-\mathrm{NMR}\left(\mathrm{CDCl}_{3}\right): \delta 8.46(\mathrm{~s}, 1 \mathrm{H}, \mathrm{Py}-\mathrm{H}), 7.91(\mathrm{~d}, J=8.4 \mathrm{~Hz}, 1 \mathrm{H}, \mathrm{Py}-\mathrm{H})$, $7.85(\mathrm{~s}, 1 \mathrm{H}, \mathrm{CH}=\mathrm{N}), 7.36(\mathrm{~d}, J=7.6 \mathrm{~Hz}, 2 \mathrm{H}, \mathrm{Ar}-\mathrm{H}$ and $\mathrm{Py}-\mathrm{H}), 7.23-7.27(\mathrm{~m}, 1 \mathrm{H}, \mathrm{ArH}), 7.01-7.13(\mathrm{~m}$, 
$4 \mathrm{H}, \mathrm{ArH}), 6.93(\mathrm{~s}, 1 \mathrm{H}, \mathrm{Ar}-\mathrm{H}), 6.80(\mathrm{~d}, J=8.4 \mathrm{~Hz}, 1 \mathrm{H}, \mathrm{Ar}-\mathrm{H}), 5.02\left(\mathrm{~s}, 2 \mathrm{H}, \mathrm{CH}_{2}\right), 3.63\left(\mathrm{~s}, 3 \mathrm{H}, \mathrm{N}-\mathrm{CH}_{3}\right)$, $2.39\left(\mathrm{~s}, 3 \mathrm{H}, \mathrm{CH}_{3}\right) .{ }^{13} \mathrm{C}-\mathrm{NMR}\left(\mathrm{CDCl}_{3}\right): \delta 165.8,157.2,152.8,147.1,146.9,145.5,145.4,140.4,136.7$, 135.5, 134.9, 130.8, 130.1, 125.1, 124.0, 121.3, 116.0, 113.6, 111.3, 100.4, 75.5, 34.3, 14.5. Anal. Calcd for $\mathrm{C}_{25} \mathrm{H}_{20} \mathrm{ClF}_{3} \mathrm{~N}_{4} \mathrm{O}_{3}$ : C 58.09; H 3.90; N 10.84. Found: C 58.18; H 3.81; N 10.72.

1,3-Dimethyl-5-(4-chlorophenoxy)-1H-pyrazole-4-carbaldehyde-O-[4-(5-trifluoromethylpyridin-2-yloxy)phenylmethyl] -oxime (8i). White solid, yield 58\%, mp 67-69 ${ }^{\circ} \mathrm{C} .{ }^{1} \mathrm{H}-\mathrm{NMR}\left(\mathrm{CDCl}_{3}\right): \delta 8.46(\mathrm{~s}, 1 \mathrm{H}, \mathrm{Py}-\mathrm{H}), 7.91(\mathrm{~d}$, $J=8.4 \mathrm{~Hz}, 1 \mathrm{H}, \mathrm{Py}-\mathrm{H}), 7.83(\mathrm{~s}, 1 \mathrm{H}, \mathrm{CH}=\mathrm{N}), 7.35(\mathrm{~d}, J=8.0 \mathrm{~Hz}, 2 \mathrm{H}, \mathrm{Ar}-\mathrm{H}$ and Py-H), $7.29(\mathrm{~d}, J=8.4 \mathrm{~Hz}$, 2H, Ar-H), $7.12(\mathrm{~d}, J=7.6 \mathrm{~Hz}, 2 \mathrm{H}, \mathrm{Ar}-\mathrm{H}), 7.03(\mathrm{~d}, J=8.4 \mathrm{~Hz}, 1 \mathrm{H}, \mathrm{Ar}-\mathrm{H}), 6.85(\mathrm{~d}, J=8.4 \mathrm{~Hz}, 2 \mathrm{H}, \mathrm{Ar}-\mathrm{H})$, $5.02\left(\mathrm{~s}, 2 \mathrm{H}, \mathrm{CH}_{2}\right), 3.62\left(\mathrm{~s}, 3 \mathrm{H}, \mathrm{N}-\mathrm{CH}_{3}\right), 2.38\left(\mathrm{~s}, 3 \mathrm{H}, \mathrm{CH}_{3}\right) .{ }^{13} \mathrm{C}-\mathrm{NMR}\left(\mathrm{CDCl}_{3}\right): \delta 165.8,155.2,152.8,147.2$, 147.0, 145.5, 145.4, 140.5, 136.7, 134.9, 130.0, 129.9, 128.7, 121.3, 119.2, 116.6, 111.4, 100.3, 75.5, 34.2, 14.5. Anal. Calcd for $\mathrm{C}_{25} \mathrm{H}_{20} \mathrm{ClF}_{3} \mathrm{~N}_{4} \mathrm{O}_{3}$ : C 58.09; H 3.90; N 10.84. Found: C 57.93; H 4.06; N 10.98.

1,3-Dimethyl-5-(2-bromophenoxy)-1H-pyrazole-4-carbaldehyde-O-[4-(5-trifluoromethylpyridin-2-yloxy)phenylmethyl] -oxime (8j). White oil, yield 47\%. ${ }^{1} \mathrm{H}-\mathrm{NMR}\left(\mathrm{CDCl}_{3}\right): \delta 8.46(\mathrm{~s}, 1 \mathrm{H}, \mathrm{Py}-\mathrm{H}), 7.91(\mathrm{~d}, J=8.4 \mathrm{~Hz}, 1 \mathrm{H}$, Py-H), $7.82(\mathrm{~s}, 1 \mathrm{H}, \mathrm{CH}=\mathrm{N}), 7.64(\mathrm{~d}, J=8.0 \mathrm{~Hz}, 1 \mathrm{H}, \mathrm{Ar}-\mathrm{H}), 7.36(\mathrm{~d}, J=7.6 \mathrm{~Hz}, 2 \mathrm{H}, \mathrm{Ar}-\mathrm{H}$ and Py-H), 7.10-7.24 (m, 3H, ArH), $7.02(\mathrm{~d}, J=8.0 \mathrm{~Hz}, 2 \mathrm{H}, \mathrm{Ar}-\mathrm{H}), 6.68(\mathrm{~d}, J=8.0 \mathrm{~Hz}, 1 \mathrm{H}, \mathrm{Ar}-\mathrm{H}), 5.01\left(\mathrm{~s}, 2 \mathrm{H}, \mathrm{CH}_{2}\right)$, $3.66\left(\mathrm{~s}, 3 \mathrm{H}, \mathrm{N}-\mathrm{CH}_{3}\right), 2.39\left(\mathrm{~s}, 3 \mathrm{H}, \mathrm{CH}_{3}\right) .{ }^{13} \mathrm{C}-\mathrm{NMR}\left(\mathrm{CDCl}_{3}\right): \delta 165.8,153.1,152.8,147.2,147.0,145.5$, 140.3, 136.7, 134.0, 130.1, 128.8, 125.0, 121.3, 115.4, 111.4, 111.3, 100.2, 75.5, 34.3, 14.5. Anal. Calcd for $\mathrm{C}_{25} \mathrm{H}_{20} \mathrm{BrF}_{3} \mathrm{~N}_{4} \mathrm{O}_{3}$ : C 53.49; H 3.59; N 9.98. Found: C 53.62; H 3.40; N 9.80.

1,3-Dimethyl-5-(3-bromophenoxy)-1H-pyrazole-4-carbaldehyde-O-[4-(5-trifluoromethylpyridin-2-yloxy)phenylmethyl] -oxime (8k). White oil, yield 50\%. ${ }^{1} \mathrm{H}-\mathrm{NMR}\left(\mathrm{CDCl}_{3}\right): \delta 8.46(\mathrm{~s}, 1 \mathrm{H}, \mathrm{Py}-\mathrm{H}), 7.91(\mathrm{~d}, J=8.4 \mathrm{~Hz}, 1 \mathrm{H}, \mathrm{Py}-\mathrm{H})$, $7.85(\mathrm{~s}, 1 \mathrm{H}, \mathrm{CH}=\mathrm{N}), 7.36(\mathrm{~d}, J=8.0 \mathrm{~Hz}, 2 \mathrm{H}, \mathrm{Ar}-\mathrm{H}$ and Py-H), $7.26(\mathrm{~d}, J=7.6 \mathrm{~Hz}, 1 \mathrm{H}, \mathrm{Ar}-\mathrm{H}), 7.09-7.21$ $(\mathrm{m}, 4 \mathrm{H}, \mathrm{ArH}), 7.02(\mathrm{~d}, J=8.4 \mathrm{~Hz}, 1 \mathrm{H}, \mathrm{Ar}-\mathrm{H}), 6.85(\mathrm{~d}, J=8.0 \mathrm{~Hz}, 1 \mathrm{H}, \mathrm{Ar}-\mathrm{H}), 5.02\left(\mathrm{~s}, 2 \mathrm{H}, \mathrm{CH}_{2}\right), 3.63(\mathrm{~s}$, $\left.3 \mathrm{H}, \mathrm{N}-\mathrm{CH}_{3}\right), 2.39\left(\mathrm{~s}, 3 \mathrm{H}, \mathrm{CH}_{3}\right) .{ }^{13} \mathrm{C}-\mathrm{NMR}\left(\mathrm{CDCl}_{3}\right): \delta 165.8,157.2,152.8,147.1,146.8,145.5,145.4,140.4$, 136.7, 134.9, 131.1, 130.0, 126.9, 125.0, 123.2, 121.3, 118.9, 114.0, 111.3, 100.4, 75.5, 34.3, 14.5. Anal. Calcd for $\mathrm{C}_{25} \mathrm{H}_{20} \mathrm{BrF}_{3} \mathrm{~N}_{4} \mathrm{O}_{3}$ : C 53.49; H 3.59; N 9.98. Found: C 53.38; H 3.72; N 10.13.

1,3-Dimethyl-5-(4-bromophenoxy)-1H-pyrazole-4-carbaldehyde-O-[4-(5-trifluoromethylpyridin-2-yloxy)phenylmethyl] -oxime (81). White solid, yield 53\%, mp, 68-70 ${ }^{\circ} \mathrm{C} .{ }^{1} \mathrm{H}-\mathrm{NMR}\left(\mathrm{CDCl}_{3}\right): \delta 8.46(\mathrm{~s}, 1 \mathrm{H}, \mathrm{Py}-\mathrm{H}), 7.92(\mathrm{~d}$, $J=8.8 \mathrm{~Hz}, 1 \mathrm{H}, \mathrm{Py}-\mathrm{H}), 7.84(\mathrm{~s}, 1 \mathrm{H}, \mathrm{CH}=\mathrm{N}), 7.43(\mathrm{~d}, J=7.2 \mathrm{~Hz}, 2 \mathrm{H}, \mathrm{Ar}-\mathrm{H}), 7.35(\mathrm{~d}, J=7.2 \mathrm{~Hz}, 2 \mathrm{H}, \mathrm{Ar}-\mathrm{H}$ and Py-H), $7.12(\mathrm{~d}, J=7.2 \mathrm{~Hz}, 2 \mathrm{H}, \mathrm{Ar}-\mathrm{H}), 7.03(\mathrm{~d}, J=8.8 \mathrm{~Hz}, 1 \mathrm{H}, \mathrm{Ar}-\mathrm{H}), 6.80(\mathrm{~d}, J=7.2 \mathrm{~Hz}, 2 \mathrm{H}, \mathrm{Ar}-\mathrm{H})$, $5.02\left(\mathrm{~s}, 2 \mathrm{H}, \mathrm{CH}_{2}\right), 3.63\left(\mathrm{~s}, 3 \mathrm{H}, \mathrm{N}-\mathrm{CH}_{3}\right), 2.38\left(\mathrm{~s}, 3 \mathrm{H}, \mathrm{CH}_{3}\right) .{ }^{13} \mathrm{C}-\mathrm{NMR}\left(\mathrm{CDCl}_{3}\right): \delta 165.8,155.8,152.8,147.1$, 145.5, 145.4, 140.4, 136.7, 136.6, 134.9, 132.9, 130.1, 121.3, 117.1, 116.1, 111.4, 109.2, 100.3, 75.5, 34.2, 14.5. Anal. Calcd for $\mathrm{C}_{25} \mathrm{H}_{20} \mathrm{BrF}_{3} \mathrm{~N}_{4} \mathrm{O}_{3}$ : C 53.49; H 3.59; N 9.98. Found: $\mathrm{C} 53.32 ; \mathrm{H} 3.76 ; \mathrm{N} 10.09$.

1,3-Dimethyl-5-(4-iodophenoxy)-1H-pyrazole-4-carbaldehyde-O-[4-(5-trifluoromethylpyridin-2-yloxy)phenylmethyl] -oxime (8m). White solid, yield 55\%, mp 59-60 ${ }^{\circ} \mathrm{C} .{ }^{1} \mathrm{H}-\mathrm{NMR}\left(\mathrm{CDCl}_{3}\right): \delta 8.46(\mathrm{~s}, 1 \mathrm{H}, \mathrm{Py}-\mathrm{H}), 7.91(\mathrm{~d}$, $J=8.8 \mathrm{~Hz}, 1 \mathrm{H}, \mathrm{Py}-\mathrm{H}), 7.83(\mathrm{~s}, 1 \mathrm{H}, \mathrm{CH}=\mathrm{N}), 7.61(\mathrm{~d}, J=7.2 \mathrm{~Hz}, 2 \mathrm{H}, \mathrm{Ar}-\mathrm{H}), 7.35(\mathrm{~d}, J=7.6 \mathrm{~Hz}, 2 \mathrm{H}, \mathrm{Ar}-\mathrm{H}$ and Py-H), $7.12(\mathrm{~d}, J=6.8 \mathrm{~Hz}, 2 \mathrm{H}, \mathrm{Ar}-\mathrm{H}), 7.03(\mathrm{~d}, J=8.4 \mathrm{~Hz}, 1 \mathrm{H}, \mathrm{Ar}-\mathrm{H}), 6.69(\mathrm{~d}, J=7.2 \mathrm{~Hz}, 2 \mathrm{H}, \mathrm{Ar}-\mathrm{H})$, $5.01\left(\mathrm{~s}, 2 \mathrm{H}, \mathrm{CH}_{2}\right), 3.61\left(\mathrm{~s}, 3 \mathrm{H}, \mathrm{N}-\mathrm{CH}_{3}\right), 2.38\left(\mathrm{~s}, 3 \mathrm{H}, \mathrm{CH}_{3}\right) .{ }^{13} \mathrm{C}-\mathrm{NMR}\left(\mathrm{CDCl}_{3}\right): \delta 165.8,156.6,152.8,147.0$, 146.9, 145.5, 145.4, 140.4, 138.8, 136.7, 134.9, 130.1, 125.1, 121.3, 117.6, 111.4, 100.3, 86.4, 75.5, 34.2, 14.5 . Anal. Calcd for $\mathrm{C}_{25} \mathrm{H}_{20} \mathrm{~F}_{3} \mathrm{IN}_{4} \mathrm{O}_{3}$ : C 49.36; H 3.31; N 9.21. Found: C 49.51; H 3.16; N 9.03.

1,3-Dimethyl-5-phenoxy-1H-pyrazole-4-carbaldehyde-O-[4-(5-trifluoromethylpyridin-2-yloxy)phenylmethyl]oxime (8n). White solid, yield 56\%, mp 45-46 ${ }^{\circ} \mathrm{C} .{ }^{1} \mathrm{H}-\mathrm{NMR}\left(\mathrm{CDCl}_{3}\right): \delta 8.46(\mathrm{~s}, 1 \mathrm{H}, \mathrm{Py}-\mathrm{H}), 7.91(\mathrm{~d}$, $J=8.4 \mathrm{~Hz}, 1 \mathrm{H}, \mathrm{Py}-\mathrm{H}), 7.84(\mathrm{~s}, 1 \mathrm{H}, \mathrm{CH}=\mathrm{N}), 7.34-7.39(\mathrm{~m}, 4 \mathrm{H}, \mathrm{ArH}$ and $\mathrm{Py}-\mathrm{H}), 7.01-7.12(\mathrm{~m}, 4 \mathrm{H}, \mathrm{ArH})$, $6.92(\mathrm{~d}, J=7.6 \mathrm{~Hz}, 2 \mathrm{H}, \mathrm{Ar}-\mathrm{H}), 5.03\left(\mathrm{~s}, 2 \mathrm{H}, \mathrm{CH}_{2}\right), 3.63\left(\mathrm{~s}, 3 \mathrm{H}, \mathrm{N}_{-} \mathrm{CH}_{3}\right), 2.40\left(\mathrm{~s}, 3 \mathrm{H}, \mathrm{CH}_{3}\right) .{ }^{13} \mathrm{C}-\mathrm{NMR}$ $\left(\mathrm{CDCl}_{3}\right): \delta 165.8,156.8,152.8,147.8,146.9,145.5,145.4,140.8,136.7,135.0,130.1,130.0,125.1,123.7$, $121.3,115.3,111.3,100.3,75.5,34.2,14.8$. Anal. Calcd for $\mathrm{C}_{25} \mathrm{H}_{21} \mathrm{~F}_{3} \mathrm{~N}_{4} \mathrm{O}_{3}: \mathrm{C} 62.24 ; \mathrm{H} 4.39 ; \mathrm{N} 11.61$. Found: C 62.41; H 4.23; N 11.42 . 
1,3-Dimethyl-5-(2-methoxyphenoxy)-1H-pyrazole-4-carbaldehyde-O-[4-(5-trifluoromethylpyridin-2-yloxy)phenylmethyl] -oxime (8o). White solid, yield 52\%, mp 55-57 ${ }^{\circ} \mathrm{C} .{ }^{1} \mathrm{H}-\mathrm{NMR}\left(\mathrm{CDCl}_{3}\right): \delta 8.45(\mathrm{~s}, 1 \mathrm{H}, \mathrm{Py}-\mathrm{H}), 7.90(\mathrm{~d}$, $J=8.8 \mathrm{~Hz}, 1 \mathrm{H}, \mathrm{Py}-\mathrm{H}), 7.80(\mathrm{~s}, 1 \mathrm{H}, \mathrm{CH}=\mathrm{N}), 7.38(\mathrm{~d}, J=8.0 \mathrm{~Hz}, 2 \mathrm{H}, \mathrm{Ar}-\mathrm{H}$ and Py-H), 7.00-7.12 (m, 5H, ArH), 6.71-6.88 (m, 2H, ArH), $5.03\left(\mathrm{~s}, 2 \mathrm{H}, \mathrm{CH}_{2}\right), 3.91\left(\mathrm{~s}, 3 \mathrm{H}, \mathrm{OCH}_{3}\right), 3.65\left(\mathrm{~s}, 3 \mathrm{H}, \mathrm{N}-\mathrm{CH}_{3}\right), 2.38(\mathrm{~s}, 3 \mathrm{H}$, $\left.\mathrm{CH}_{3}\right) .{ }^{13} \mathrm{C}-\mathrm{NMR}\left(\mathrm{CDCl}_{3}\right): \delta 165.8,152.7,149.0,148.5,146.8,145.8,145.5,145.4,140.9,136.7,136.6,135.1$, $130.1,124.6,121.3,121.0,115.9,112.8,111.3,99.6,75.4,56.1,34.2,14.9$. Anal. Calcd for $\mathrm{C}_{26} \mathrm{H}_{23} \mathrm{~F}_{3} \mathrm{~N}_{4} \mathrm{O}_{4}$ : C 60.93; H 4.52; N 10.93. Found: C 60.78; H 4.71; N 11.06.

1,3-Dimethyl-5-(4-methoxyphenoxy)-1H-pyrazole-4-carbaldehyde-O-[4-(5-trifluoromethylpyridin-2-yloxy)phenylmethyl] -oxime (8p). White solid, yield 63\%, mp 76-78 ${ }^{\circ} \mathrm{C} .{ }^{1} \mathrm{H}-\mathrm{NMR}\left(\mathrm{CDCl}_{3}\right): \delta 8.45(\mathrm{~s}, 1 \mathrm{H}, \mathrm{Py}-\mathrm{H}), 7.90(\mathrm{~d}$, $J=8.8 \mathrm{~Hz}, 1 \mathrm{H}, \mathrm{Py}-\mathrm{H}), 7.82(\mathrm{~s}, 1 \mathrm{H}, \mathrm{CH}=\mathrm{N}), 7.39(\mathrm{~d}, J=8.4 \mathrm{~Hz}, 2 \mathrm{H}, \mathrm{Ar}-\mathrm{H}$ and Py-H), $7.11(\mathrm{~d}, J=8.4 \mathrm{~Hz}$, 2H, Ar-H), 7.01 (d, J = 8.4 Hz, 1H, Ar-H), 6.85 (s, 4H, Ar-H), 5.04 (s, 2H, CH 2$), 3.77$ (s, 3H, OCH $), 3.62$ $\left(\mathrm{s}, 3 \mathrm{H}, \mathrm{N}-\mathrm{CH}_{3}\right), 2.38\left(\mathrm{~s}, 3 \mathrm{H}, \mathrm{CH}_{3}\right) .{ }^{13} \mathrm{C}-\mathrm{NMR}\left(\mathrm{CDCl}_{3}\right): \delta 165.8,155.8,152.8,150.7,148.5,146.9,145.5$, $145.4,140.9,136.7,136.6,135.0,130.1,121.3,116.4,114.9,111.3,99.9,75.4,55.7,34.2,14.8$. Anal. Calcd for $\mathrm{C}_{26} \mathrm{H}_{23} \mathrm{~F}_{3} \mathrm{~N}_{4} \mathrm{O}_{4}$ : C 60.93; $\mathrm{H} 4.52 ; \mathrm{N} 10.93$. Found: C 61.04; $\mathrm{H} 4.35 ; \mathrm{N} 10.78$.

1,3-Dimethyl-5-(4-methylphenoxy)-1H-pyrazole-4-carbaldehyde-O-[4-(5-trifluoromethylpyridin-2-yloxy)phenylmethyl] -oxime (8q). White solid, yield 61\%, mp 52-53 ${ }^{\circ} \mathrm{C} .{ }^{1} \mathrm{H}-\mathrm{NMR}\left(\mathrm{CDCl}_{3}\right): \delta 8.46(\mathrm{~s}, 1 \mathrm{H}, \mathrm{Py}-\mathrm{H}), 7.90(\mathrm{~d}$, $J=8.4 \mathrm{~Hz}, 1 \mathrm{H}, \mathrm{Py}-\mathrm{H}), 7.84(\mathrm{~s}, 1 \mathrm{H}, \mathrm{CH}=\mathrm{N}), 7.39(\mathrm{~d}, J=7.6 \mathrm{~Hz}, 2 \mathrm{H}, \mathrm{Ar}-\mathrm{H}$ and Py-H), $7.12(\mathrm{~d}, J=7.2 \mathrm{~Hz}$, $4 \mathrm{H}, \mathrm{Ar}-\mathrm{H}), 7.01$ (d, J = 8.4 Hz, 1H, Ar-H), 6.81 (d, J = 7.6 Hz, 2H, Ar-H), 5.05 (s, 2H, CH $), 3.61$ (s, 3H, $\left.\mathrm{N}-\mathrm{CH}_{3}\right), 2.40\left(\mathrm{~s}, 3 \mathrm{H}, \mathrm{CH}_{3}\right), 2.32\left(\mathrm{~s}, 3 \mathrm{H}, \mathrm{CH}_{3}\right) .{ }^{13} \mathrm{C}-\mathrm{NMR}\left(\mathrm{CDCl}_{3}\right): \delta 165.8,154.8,152.8,148.1,146.8$, 145.5, 145.4, 140.9, 136.7, 136.6, 135.1, 133.1, 130.4, 130.1, 121.3, 115.1, 111.3, 100.1, 75.4, 34.2, 20.5, 14.9. Anal. Calcd for $\mathrm{C}_{26} \mathrm{H}_{23} \mathrm{~F}_{3} \mathrm{~N}_{4} \mathrm{O}_{3}$ : C 62.90; $\mathrm{H}$ 4.67; N 11.28. Found: C 62.73; H 4.81; N 11.42.

1,3-Dimethyl-5-(4-tert-butylphenoxy)-1H-pyrazole-4-carbaldehyde-O-[4-(5-trifluoromethylpyridin-2-yloxy)phenylmethyl] -oxime (8r). White oil, yield 57\%. ${ }^{1} \mathrm{H}-\mathrm{NMR}\left(\mathrm{CDCl}_{3}\right)$ : $\delta 8.46(\mathrm{~s}, 1 \mathrm{H}, \mathrm{Py}-\mathrm{H}), 7.91(\mathrm{~d}, J=8.8 \mathrm{~Hz}, 1 \mathrm{H}, \mathrm{Py}-\mathrm{H})$, $7.85(\mathrm{~s}, 1 \mathrm{H}, \mathrm{CH}=\mathrm{N}), 7.41(\mathrm{~d}, J=8.0 \mathrm{~Hz}, 2 \mathrm{H}, \mathrm{Ar}-\mathrm{H}$ and Py-H), $7.12(\mathrm{~d}, J=8.4 \mathrm{~Hz}, 2 \mathrm{H}, \mathrm{Ar}-\mathrm{H}), 7.02$ (d, $J=8.8 \mathrm{~Hz}, 1 \mathrm{H}, \mathrm{Ar}-\mathrm{H}), 6.84(\mathrm{~d}, J=8.4 \mathrm{~Hz}, 2 \mathrm{H}, \mathrm{Ar}-\mathrm{H}), 5.05\left(\mathrm{~s}, 2 \mathrm{H}, \mathrm{CH}_{2}\right), 3.62\left(\mathrm{~s}, 3 \mathrm{H}, \mathrm{N}-\mathrm{CH}_{3}\right), 2.41(\mathrm{~s}, 3 \mathrm{H}$, $\left.\mathrm{CH}_{3}\right), 1.32\left(\mathrm{~s}, 9 \mathrm{H}, t-\mathrm{C}_{4} \mathrm{H}_{9}\right) .{ }^{13} \mathrm{C}-\mathrm{NMR}\left(\mathrm{CDCl}_{3}\right): \delta 165.8,154.6,152.8,148.1,146.8,145.5,145.4,141.0$, 136.7, 136.6, 135.0, 130.1, 126.8, 121.3, 100.2, 75.4, 34.3, 34.2, 31.4, 14.9. Anal. Calcd for $\mathrm{C}_{29} \mathrm{H}_{29} \mathrm{~F}_{3} \mathrm{~N}_{4} \mathrm{O}_{3}$ : C 64.67; H 5.43; N 10.40. Found: C 64.52; H 5.61; N 10.58.

1,3-Dimethyl-5-(4-trifluoromethoxyphenoxy)-1H-pyrazole-4-carbaldehyde-O-[4-(5-trifluoromethylpyridin-2yloxy)phenylmethyl]-oxime (8s). White solid, yield 52\%, mp $75-77^{\circ} \mathrm{C} .{ }^{1} \mathrm{H}-\mathrm{NMR}\left(\mathrm{CDCl}_{3}\right): \delta 8.45(\mathrm{~s}, 1 \mathrm{H}$, Py-H), 7.91 (d, J = 8.4 Hz, 1H, Py-H), $7.85(\mathrm{~s}, 1 \mathrm{H}, \mathrm{CH}=\mathrm{N}), 7.36$ (d, J = 8.0 Hz, 2H, Ar-H and Py-H), $7.19(\mathrm{~d}, J=8.4 \mathrm{~Hz}, 2 \mathrm{H}, \mathrm{Ar}-\mathrm{H}), 7.11(\mathrm{~d}, J=8.0 \mathrm{~Hz}, 2 \mathrm{H}, \mathrm{Ar}-\mathrm{H}), 7.02(\mathrm{~d}, J=8.4 \mathrm{~Hz}, 1 \mathrm{H}, \mathrm{Ar}-\mathrm{H}), 6.92(\mathrm{~d}$, $J=8.8 \mathrm{~Hz}, 2 \mathrm{H}, \mathrm{Ar}-\mathrm{H}), 5.00\left(\mathrm{~s}, 2 \mathrm{H}, \mathrm{CH}_{2}\right), 3.64\left(\mathrm{~s}, 3 \mathrm{H}, \mathrm{N}-\mathrm{CH}_{3}\right), 2.39\left(\mathrm{~s}, 3 \mathrm{H}, \mathrm{CH}_{3}\right) .{ }^{13} \mathrm{C}-\mathrm{NMR}\left(\mathrm{CDCl}_{3}\right)$ : $\delta$ 154.9, 152.8, 147.1, 145.5, 140.4, 136.7, 134.8, 130.0, 122.8, 121.3, 116.4, 111.4, 100.3, 75.5, 34.2, 14.5. Anal. Calcd for $\mathrm{C}_{26} \mathrm{H}_{20} \mathrm{~F}_{6} \mathrm{~N}_{4} \mathrm{O}_{4}$ : C 55.13; H 3.56; N 9.89. Found: C 55.01; H 3.71; N 10.03.

1,3-Dimethyl-5-(2,3-difluorophenoxy)-1H-pyrazole-4-carbaldehyde-O-[4-(5-trifluoromethylpyridin-2-yloxy) phenylmethyl]-oxime (8t). White solid, yield 44\%, mp 61-62 ${ }^{\circ} \mathrm{C} .{ }^{1} \mathrm{H}-\mathrm{NMR}\left(\mathrm{CDCl}_{3}\right): \delta 8.45(\mathrm{~s}, 1 \mathrm{H}, \mathrm{Py}-\mathrm{H})$, $7.91(\mathrm{~d}, J=8.8 \mathrm{~Hz}, 1 \mathrm{H}, \mathrm{Py}-\mathrm{H}), 7.85(\mathrm{~s}, 1 \mathrm{H}, \mathrm{CH}=\mathrm{N}), 7.36(\mathrm{~d}, J=8.0 \mathrm{~Hz}, 2 \mathrm{H}, \mathrm{Ar}-\mathrm{H}$ and Py-H), 7.11 (d, $J=8.0 \mathrm{~Hz}, 2 \mathrm{H}, \mathrm{Ar}-\mathrm{H}), 7.02(\mathrm{~d}, J=8.4 \mathrm{~Hz}, 1 \mathrm{H}, \mathrm{Ar}-\mathrm{H}), 6.93-6.97(\mathrm{~m}, 2 \mathrm{H}, \mathrm{ArH}), 6.54(\mathrm{~d}, J=8.4 \mathrm{~Hz}, 1 \mathrm{H}$, Ar-H), 5.00 (s, 2H, CH $), 3.68\left(\mathrm{~s}, 3 \mathrm{H}, \mathrm{N}-\mathrm{CH}_{3}\right), 2.36\left(\mathrm{~s}, 3 \mathrm{H}, \mathrm{CH}_{3}\right) .{ }^{13} \mathrm{C}-\mathrm{NMR}\left(\mathrm{CDCl}_{3}\right): \delta 165.8,152.8,147.1$, 146.7, 145.5, 145.4, 140.1, 136.7, 136.6, 134.9, 129.9, 123.5, 123.4, 121.3, 112.3, 112.1, 111.6, 111.5, 111.3, 100.0, 75.5, 34.2, 14.3. Anal. Calcd for $\mathrm{C}_{25} \mathrm{H}_{19} \mathrm{~F}_{5} \mathrm{~N}_{4} \mathrm{O}_{3}$ : C 57.92; H 3.69; N 10.81. Found: C 57.76; H 3.83; N 10.96.

1,3-Dimethyl-5-(2,4-dichlorophenoxy)-1H-pyrazole-4-carbaldehyde-O-[4-(5-trifluoromethylpyridin-2-yloxy) phenylmethyl]-oxime (8u). White solid, yield 52\%, mp $109-111{ }^{\circ} \mathrm{C} .{ }^{1} \mathrm{H}-\mathrm{NMR}\left(\mathrm{CDCl}_{3}\right): \delta 8.45(\mathrm{~s}, 1 \mathrm{H}$, Py-H), 7.90 (d, J = 8.4 Hz, 1H, Py-H), 7.82 (s, 1H, CH=N), 7.46 (s, 1H, Ar-H), 7.33 (d, J = 8.4 Hz, 2H, Ar-H and Py-H), 7.00-7.12 (m, 4H, ArH), $6.63(\mathrm{~d}, J=8.8 \mathrm{~Hz}, 2 \mathrm{H}, \mathrm{Ar}-\mathrm{H}), 4.98\left(\mathrm{~s}, 2 \mathrm{H}, \mathrm{CH}_{2}\right), 3.65(\mathrm{~s}, 3 \mathrm{H}$, $\left.\mathrm{N}-\mathrm{CH}_{3}\right), 2.36\left(\mathrm{~s}, 3 \mathrm{H}, \mathrm{CH}_{3}\right) .{ }^{13} \mathrm{C}-\mathrm{NMR}\left(\mathrm{CDCl}_{3}\right): \delta 165.8,152.8,150.9,147.1,146.5,145.5,145.4,140.1$, 
136.7, 134.9, 130.5, 130.0, 129.0, 127.9, 125.1, 123.6, 122.4, 121.3, 116.3, 111.4, 100.1, 75.5, 34.2, 14.2. Anal. Calcd for $\mathrm{C}_{25} \mathrm{H}_{19} \mathrm{Cl}_{2} \mathrm{~F}_{3} \mathrm{~N}_{4} \mathrm{O}_{3}$ : C 54.46; H 3.47; N 10.16. Found: C 54.62; H 3.29; N 10.02.

1,3-Dimethyl-5-(2,3-dimethylphenoxy)-1H-pyrazole-4-carbaldehyde-O-[4-(5-trifluoromethylpyridin-2-yloxy) phenylmethyl]-oxime (8v). White oil, yield 49\%. ${ }^{1} \mathrm{H}-\mathrm{NMR}\left(\mathrm{CDCl}_{3}\right): \delta 8.46(\mathrm{~s}, 1 \mathrm{H}, \mathrm{Py}-\mathrm{H}), 7.91(\mathrm{~d}$, $J=8.0 \mathrm{~Hz}, 1 \mathrm{H}, \mathrm{Py}-\mathrm{H}), 7.77(\mathrm{~s}, 1 \mathrm{H}, \mathrm{CH}=\mathrm{N}), 7.38(\mathrm{~d}, J=8.0 \mathrm{~Hz}, 2 \mathrm{H}, \mathrm{Ar}-\mathrm{H}$ and Py-H), $7.12(\mathrm{~d}, J=8.0 \mathrm{~Hz}$, 2H, Ar-H), 6.93-7.03 (m, 3H, ArH), 6.42 (d, J = 8.0 Hz, 1H, Ar-H), $5.04\left(\mathrm{~s}, 2 \mathrm{H}, \mathrm{CH}_{2}\right), 3.62\left(\mathrm{~s}, 3 \mathrm{H}, \mathrm{N}-\mathrm{CH}_{3}\right)$, $2.41\left(\mathrm{~s}, 3 \mathrm{H}, \mathrm{CH}_{3}\right), 2.35\left(\mathrm{~s}, 3 \mathrm{H}, \mathrm{CH}_{3}\right), 2.32\left(\mathrm{~s}, 3 \mathrm{H}, \mathrm{CH}_{3}\right) .{ }^{13} \mathrm{C}-\mathrm{NMR}\left(\mathrm{CDCl}_{3}\right): \delta 165.8,154.9,152.8,148.6$, 146.9, 145.5, 141.0, 139.0, 136.7, 136.6, 135.1, 130.1, 126.2, 125.2, 121.3, 111.3, 111.2, 99.8, 75.4, 34.1, 20.0, 14.9, 11.8. Anal. Calcd for $\mathrm{C}_{27} \mathrm{H}_{25} \mathrm{~F}_{3} \mathrm{~N}_{4} \mathrm{O}_{3}$ : C 63.52; $\mathrm{H} 4.94 ; \mathrm{N} 10.97$. Found: C 63.36; H 5.09; N 11.05.

1,3-Dimethyl-5-(2,4-dimethylphenoxy)-1H-pyrazole-4-carbaldehyde-O-[4-(5-trifluoromethylpyridin-2-yloxy)phenylmethyl] -oxime (8w). White oil, yield 53\%. ${ }^{1} \mathrm{H}-\mathrm{NMR}\left(\mathrm{CDCl}_{3}\right): \delta 8.46(\mathrm{~s}, 1 \mathrm{H}, \mathrm{Py}-\mathrm{H}), 7.91(\mathrm{~d}, J=8.4 \mathrm{~Hz}, 1 \mathrm{H}, \mathrm{Py}-\mathrm{H})$, $7.76(\mathrm{~s}, 1 \mathrm{H}, \mathrm{CH}=\mathrm{N}), 7.38(\mathrm{~d}, J=8.0 \mathrm{~Hz}, 2 \mathrm{H}, \mathrm{Ar}-\mathrm{H}$ and $\mathrm{Py}-\mathrm{H}), 7.11(\mathrm{~d}, J=7.6 \mathrm{~Hz}, 2 \mathrm{H}, \mathrm{Ar}-\mathrm{H}), 6.88-7.05(\mathrm{~m}$, $3 \mathrm{H}, \mathrm{ArH}), 6.45$ (d, J = 8.4 Hz, 1H, Ar-H), $5.04\left(\mathrm{~s}, 2 \mathrm{H}, \mathrm{CH}_{2}\right), 3.62\left(\mathrm{~s}, 3 \mathrm{H}, \mathrm{N}-\mathrm{CH}_{3}\right), 2.40\left(\mathrm{~s}, 3 \mathrm{H}, \mathrm{CH}_{3}\right), 2.36$ (s, $\left.3 \mathrm{H}, \mathrm{CH}_{3}\right), 2.29\left(\mathrm{~s}, 3 \mathrm{H}, \mathrm{CH}_{3}\right) .{ }^{13} \mathrm{C}-\mathrm{NMR}\left(\mathrm{CDCl}_{3}\right): \delta 165.8,152.9,152.7,148.6,146.9,145.5,141.0,136.7,135.1$, 133.0, 132.2, 130.1, 127.5, 126.2, 125.1, 122.4, 121.7, 121.3, 113.4, 99.7, 75.4, 34.1, 20.5, 16.0, 14.9. Anal. Calcd for $\mathrm{C}_{27} \mathrm{H}_{25} \mathrm{~F}_{3} \mathrm{~N}_{4} \mathrm{O}_{3}$ : C 63.52; $\mathrm{H} 4.94 ; \mathrm{N} 10.97$. Found: C 63.69; H 4.78; N 10.82.

\subsection{Biological Tests}

\subsubsection{Bioassay Methods}

All bioassays were performed on representative test organisms reared in the laboratory. The bioassay was repeated in triplicate at $25 \pm 1{ }^{\circ} \mathrm{C}$. Assessments were made on a dead/alive basis, and mortality rates were corrected using Abbott's formula. For comparative purposes, the controls Fenpyroximate, Pyridalyl and Imidacloprid were evaluated under the same conditions.

\subsubsection{Acaricidal Activity against Tetranychus cinnabarinus}

The acaricidal activities against Tetranychus cinnabarinus of the designed compounds were evaluated using the reported procedure [27]. Sieva bean plants with primary leaves expanded to $10 \mathrm{~cm}$ were selected and cut back to one plant per pot. A small piece was cut from a leaf taken from the main colony and placed on each leaf of the test plants. This was done about $2 \mathrm{~h}$ before treatment to allow the mites to move over to the test plant and to lay eggs. The size of the piece was varied to obtain about 60-100 mites per leaf. At the time of the treatment, the piece of leaf used to transfer the mites was removed and discarded. The mite-infested plants were dipped in the test formulation for $3 \mathrm{~s}$ with agitation and set in the hood to dry. Plants were kept for $48 \mathrm{~h}$ before the numbers of live and dead adults were counted. Each experiment for one compound was triplicated.

\subsubsection{Insecticidal Activity against Plutella xylostella}

The insecticidal activities of the title compounds against Plutella xylostella were evaluated using the leaf disk assay [28]. First, a solution of each test sample in $N, N$-dimethylformamide at a concentration of $200 \mu \mathrm{g} / \mathrm{mL}$ was prepared and then diluted to the required concentration with water. Cabbage leaves were dipped into the obtained solutions for 2-3 s. After air-drying, the soaked leaves were put into a 10-cm-long tube, inoculated with second Plutella xylostella larva. Covered with gauze and then kept in a room for normal cultivation. Mortality was assessed $48 \mathrm{~h}$ after treatment. Each experiment for one compound was triplicated.

\subsubsection{Insecticidal Activity against Aphis craccivora}

Insecticidal activities of the target compounds were tested against Aphis craccivora by foliar application [29]. About 60 aphids were transferred to the shoot with 3-5 fresh leaves of horsebean. The shoot with aphids was cut and dipped into a required solution from $200 \mu \mathrm{g} / \mathrm{mL}$ to $100 \mu \mathrm{g} / \mathrm{mL}$ of 
the tested compound for $2 \mathrm{~s}$. After removing extra solutions on the leaf, the aphids were raised in the shoot at $25{ }^{\circ} \mathrm{C}$ and $85 \%$ relative humidity for $48 \mathrm{~h}$. Each experiment for one compound was triplicated.

\section{Conclusions}

In summary, 23 pyrazole oxime compounds bearing a 5-trifluoromethyl pyridyl subunit were synthesized. A preliminary evaluation of the acaricidal and insecticidal activities of the designed compounds was conducted. Most of them exhibited obvious acaricidal activity against $T$. cinnabarinus at a concentration of $200 \mu \mathrm{g} / \mathrm{mL}$, and some derivatives such as compounds $\mathbf{8 e}, \mathbf{8 f}, \mathbf{8 1}, \mathbf{8 m}, \mathbf{8 n}, \mathbf{8 p}$, and $\mathbf{8 q}$ still possessed excellent acaricidal activity against T. cinnabarinus under the concentration of $10 \mu \mathrm{g} / \mathrm{mL}$. Additionally, some compounds showed potent insecticidal activities against $P$. xylostella and A. craccivora at a concentration of $200 \mu \mathrm{g} / \mathrm{mL}$. Notably, compounds $\mathbf{8 e}$ and $\mathbf{8 1}$ were more active against $P$. xylostella than other compounds, even when the concentration was decreased to $50 \mu \mathrm{g} / \mathrm{mL}$. Among these compounds, compounds $\mathbf{8 e}, \mathbf{8} \mathbf{i}$, and $\mathbf{8 n}$ showed broad spectrum biological activities; they displayed potential insecticidal activity against $P$. xylostella and A. craccivora and beyond satisfactory acaricidal activity against $T$. cinnabarinus. Further investigations on the structural optimization and bioactivities of these pyrazole oximes are currently in progress.

Acknowledgments: This work was funded by the National Natural Science Foundation of China (Nos. 21202089, 21372135), the Research Foundation of the Six People Peak of Jiangsu Province (No. 2013-SWYY-013), the Natural Science Foundation of Jiangsu Province (No. BK20140425), the Technology Project Fund of Nantong City (Nos. CP12013002, AS2014011, MS22015020).

Author Contributions: H.H. designed the research; H.D., J.C., H.L., B.D., Y.F. performed the research and analyzed the data; Y.S. wrote the paper. All authors read and approved the final manuscript.

Conflicts of Interest: The authors declare no conflict of interest.

\section{References}

1. Lümmen, P. Complex I inhibitors as insecticides and acaricides. BBA Bioenerg. 1998, 1364, 287-296. [CrossRef]

2. Gatehouse, J.A. Biotechnological prospects for engineering insect-resistant plants. Plant Physiol. 2008, 146, 881-887. [CrossRef] [PubMed]

3. Miller, T. Control of pink bollworm. Pestic. Outlook 2001, 12, 68-70. [CrossRef]

4. Lamberth, C. Pyrazole chemistry in crop protection. Heterocycles 2007, 71, 1467-1502. [CrossRef]

5. Swanson, M.B.; Ivancic, W.A.; Saxena, A.M.; Allton, J.D.; O’Brien, G.K.; Suzuki, T.; Nishizawa, H.; Nokata, M. Direct photolysis of Fenpyroximate in a buffered aqueous solution under a xenon lamp. J. Agric. Food Chem. 1995, 43, 513-518. [CrossRef]

6. Fustero, S.; Roman, R.; Sanz-Cervera, J.F.; Simon-Fuentes, A.; Cunat, A.C.; Villanova, S.; Murguia, M. Improved regioselectivity in pyrazole formation through the use of fluorinated alcohols as solvents: Synthesis and biological activity of fluorinated Tebufenpyrad analogs. J. Org. Chem. 2008, 73, 3523-3529. [CrossRef] [PubMed]

7. Fustero, S.; Roman, R.; Sanz-Cervera, J.F.; Simon-Fuentes, A.; Bueno, J.; Villanova, S. Synthesis of new fluorinated Tebufenpyrad analogs with acaricidal activity through regioselective pyrazole formation. J. Org. Chem. 2008, 73, 8545-8552. [CrossRef] [PubMed]

8. Motoba, K.; Nishizawa, H.; Suzuki, T.; Hamaguchi, H.; Uchida, M.; Funayama, S. Species-specific detoxification metabolism of Fenpyroximate, a potent acaricide. Pestic. Biochem. Physiol. 2000, 67, 73-84. [CrossRef]

9. Kim, Y.J.; Lee, S.H.; Lee, S.W.; Ahn, Y.J. Fenpyroximate resistance in Tetranychus urticae (Acari: Tetranychidae): Cross-resistance and biochemical resistance mechanisms. Pest Manag. Sci. 2004, 60, 1001-1006. [CrossRef] [PubMed]

10. Chen, L.; Ou, X.M.; Mao, C.H.; Shang, J.; Huang, R.Q.; Bi, F.C.; Wang, Q.M. Synthesis and bioassay evaluation of 1-(4-substituteddidene-aminooxymethyl)-phenyl-3-(2,6-difluorobenzoyl)ureas. Bioorg. Med. Chem. 2007, 15, 3678-3683. [CrossRef] [PubMed]

11. Dai, H.; Xiao, Y.S.; Li, Z.; Xu, X.Y.; Qian, X.H. The thiazoylmethoxy modification on pyrazole oximes: Synthesis and insecticidal biological evaluation beyond acaricidal activity. Chin. Chem. Lett. 2014, 25, 1014-1016. [CrossRef]

12. Yang, Y.Z.; Lin, D.Y.; Fu, C.R.; Zou, X.M. Synthesis and biological evaluation of novel pyrazole oxime ether derivatives containing chlorothiazole group and pyrimidine rings. Chin. J. Org. Chem. 2015, 35, 100-108. [CrossRef] 
13. Dai, H.; Li, Y.Q.; Du, D.; Qin, X.; Zhang, X.; Yu, H.B.; Fang, J.X. Synthesis and biological activities of novel pyrazole oxime derivatives containing a 2-chloro-5-thiazolyl moiety. J. Agric. Food Chem. 2008, 56, 10805-10810. [CrossRef] [PubMed]

14. Dai, H.; Shi, L.; Zhang, H.; Li, Y.; Fang, J.; Shi, Y. Synthesis and bioactivities of novel 1-phenyl-3-methyl-5-aryloxy-1H-pyrazole-4-carbaldehyde-O-(2-chloropyridin-5-yl)methyl oximes. Chin. J. Org. Chem. 2012, 32, 1060-1066. [CrossRef]

15. Kiriyama, K.; Kagabu, S.; Nishimura, K. Insecticidal activities of the enantiomers of asymmetric 1-(6-chloro-3-pyridyl)ethyl-2-nitroiminoimidazolidine against American cockroach, cucurbit leaf beetle, green rice leafhopper and green peach aphid following injection, dipping and spraying. J. Pestic. Sci. 2004, 29, 43-45. [CrossRef]

16. Kagabu, S.; Murase, Y.; Imai, R.; Ito, N.; Nishimura, K. Effect of substituents at the 5-position of the pyridine ring of imidacloprid on insecticidal activity against Periplaneta americana. Pest Manag. Sci. 2007, 63, 75-83. [CrossRef] [PubMed]

17. Kagabu, S. Discovery of imidacloprid and further developments from strategic molecular designs. J. Agric. Food Chem. 2010, 58, 2887-2896. [CrossRef] [PubMed]

18. Lu, S.Y.; Shao, X.S.; Li, Z.; Xu, Z.P.; Zhao, S.S.; Wu, Y.L.; Xu, X.Y. Design, synthesis, and particular biological behaviors of chain-open nitromethylene neonicotinoids with cis configuration. J. Agric. Food Chem. 2012, 60, 322-330. [CrossRef] [PubMed]

19. Ye, Z.J.; Shi, L.N.; Shao, X.S.; Xu, X.Y.; Xu, Z.P.; Li, Z. Pyrrole- and dihydropyrrole-fused neonicotinoids: Design, synthesis, and insecticidal evaluation. J. Agric. Food Chem. 2013, 61, 312-319. [CrossRef] [PubMed]

20. Lu, S.Y.; Zhuang, Y.Y.; Wu, N.B.; Feng, Y.; Cheng, J.G.; Li, Z.; Chen, J.; Yuan, J.; Xu, X.Y. Synthesis and biological evaluation of nitromethylene neonicotinoids based on the enhanced conjugation. J. Agric. Food Chem. 2013, 61, 10858-10863. [CrossRef] [PubMed]

21. Xu, R.B.; Xia, R.; Luo, M.; Xu, X.Y.; Cheng, J.G.; Shao, X.S.; Li, Z. Design, synthesis, crystal structures, and insecticidal activities of eight-membered azabridge neonicotinoid analogues. J. Agric. Food Chem. 2014, 62, 381-390. [CrossRef] [PubMed]

22. Smart, B.E. Fluorine substituent effects (on bioactivity). J. Fluor. Chem. 2001, 109, 3-11. [CrossRef]

23. Begue, J.P.; Bonnet-Delpon, D.; Crousse, B.; Legros, J. The chemistry of trifluoromethyl imines and related acetals derived from fluoral. Chem. Soc. Rev. 2005, 34, 562-572. [CrossRef] [PubMed]

24. Babcock, J.M.; Gerwick, C.B.; Huang, J.X.; Loso, M.R.; Nakamura, G.; Nolting, S.P.; Rogers, R.B.; Sparks, T.C.; Thomas, J.; Watson, G.B.; et al. Biological characterization of Sulfoxaflor, a novel insecticide. Pest Manag. Sci. 2011, 67, 328-334. [CrossRef] [PubMed]

25. Sakamoto, N.; Saito, S.; Hirose, T.; Suzuki, M.; Matsuo, S.; Izumi, K.; Nagatomi, T.; Ikegami, H.; Umeda, K.; Tsushima, K.; et al. The discovery of pyridalyl: A novel insecticidal agent for controlling lepidopterous pests. Pest Manag. Sci. 2004, 60, 25-34. [CrossRef] [PubMed]

26. Park, M.S.; Park, H.J.; Park, K.H.; Lee, K.I. Introduction of N-containing heterocycles into pyrazole by nucleophilic aromatic substitution. Synth. Commun. 2004, 34, 1541-1550. [CrossRef]

27. Song, H.J.; Liu, Y.X.; Xiong, L.X.; Li, Y.Q.; Yang, N.; Wang, Q.M. Design, synthesis, and insecticidal evaluation of new pyrazole derivatives containing imine, oxime ether, and dihydroisoxazoline groups based on the inhibitor binding pocket of respiratory complex I. J. Agric. Food Chem. 2013, 61, 8730-8736. [CrossRef] [PubMed]

28. Sun, J.L.; Zhou, Y.M. Design, synthesis, and insecticidal activity of some novel diacylhydrazine and acylhydrazone derivatives. Molecules 2015, 20, 5625-5637. [CrossRef] [PubMed]

29. Shao, X.S.; Fu, H.; Xu, X.Y.; Xu, X.L.; Liu, Z.W.; Li, Z.; Qian, X.H. Divalent and oxabridged neonicotinoids constructed by dialdehydes and nitromethylene analogues of imidacloprid: Design, synthesis, crystal structure, and insecticidal activities. J. Agric. Food Chem. 2010, 58, 2696-2702. [CrossRef] [PubMed]

Sample Availability: Samples of the compounds $\mathbf{8 a - 8 w}$ are available from the authors.

(C) 2016 by the authors; licensee MDPI, Basel, Switzerland. This article is an open access article distributed under the terms and conditions of the Creative Commons by Attribution (CC-BY) license (http://creativecommons.org/licenses/by/4.0/). 